\title{
What is the influence of a reduction of planktivorous and benthivorous fish on water quality in temperate eutrophic lakes? A systematic review
}

Claes Bernes ${ }^{1 *}$, Stephen R Carpenter ${ }^{2}$, Anna Gårdmark ${ }^{3}$, Per Larsson ${ }^{4}$, Lennart Persson ${ }^{5}$, Christian Skov ${ }^{6}$, James DM Speed ${ }^{7}$ and Ellen Van Donk ${ }^{8}$

\begin{abstract}
Background: In recent decades, many attempts have been made to restore eutrophic lakes through biomanipulation. Reducing the populations of planktivorous and benthivorous fish (either directly or through stocking of piscivorous fish) may induce ecosystem changes that increase water transparency and decrease the risk of algal blooms and fish kills, at least in the short term. However, the generality of biomanipulation effects on water quality across lake types and geographical regions is not known. Therefore, we have undertaken a systematic review of such effects in eutrophic lakes in temperate regions throughout the world.
\end{abstract}

Methods: Searches for literature were made using online publication databases, search engines, specialist websites and bibliographies of literature reviews. Search terms were developed in English, Danish, Dutch and Swedish. Identified articles were screened for relevance using inclusion criteria set out in an a priori protocol. To reduce the risk of bias, we then critically appraised the combined evidence found on each biomanipulation. Data were extracted on outcomes such as Secchi depth and chlorophyll a concentration before, during and/or after manipulation, and on effect modifiers such as lake properties and amounts of fish removed or stocked.

Results: Our searches identified more than 14,500 articles. After screening for relevance, 233 of them remained. After exclusions based on critical appraisal, our evidence base included useful data on 128 biomanipulations in 123 lakes. Of these interventions, $85 \%$ had been made in Europe and 15\% in North America. Meta-analysis showed that removal of planktivores and benthivores (with or without piscivore stocking) leads to increased Secchi depth and decreased chlorophyll a concentration during intervention and the first three years afterwards. Piscivore stocking alone has no significant effect. The response of chlorophyll a levels to biomanipulation is stronger in lakes where fish removal is intense, and in lakes which are small and/or have high pre-manipulation concentrations of total phosphorus.

Conclusions: Our review improves on previous reviews of biomanipulation in that we identified a large number of case studies from many parts of the world and used a consistent, repeatable process to screen them for relevance and susceptibility to bias. Our results indicate that removal of planktivorous and benthivorous fish is a useful means of improving water quality in eutrophic lakes. Biomanipulation tends to be particularly successful in relatively small lakes with short retention times and high phosphorus levels. More thorough fish removal increases the efficacy of biomanipulation. Nonetheless successes and failures have occurred across a wide range of conditions.

Keywords: Biomanipulation, Planktivore, Benthivore, Piscivore stocking, Fish removal, Lake restoration, Eutrophication, Water quality, Phytoplankton

\footnotetext{
* Correspondence: claes.bernes@eviem.se

'Mistra Council for Evidence-Based Environmental Management, Royal Swedish

Academy of Sciences, P.O. Box 50005, SE-104 05 Stockholm, Sweden

Full list of author information is available at the end of the article
} 


\section{Background}

Over the past century, many lakes in urban or agricultural regions of the world were eutrophied due to sewage discharges or nutrient runoff from land. Excess nutrients, especially phosphorus, stimulates the growth of phytoplankton, often to such an extent that the water becomes turbid [1]. The reduced light penetration and increased sedimentation of dead planktonic algae puts submerged macrophytes at a disadvantage, in some cases even eliminating them, often with strong impacts on ecosystem interactions and dynamics [2]. Certain species of phytoplankton - cyanobacteria in particular - can give rise to massive 'algal blooms' in the summer. The decomposition of dead plankton can lead to oxygen depletion and fish kills [3].

Problems of these kinds have often persisted even when nutrient supplies from the surroundings have been reduced, e.g. through sewage treatment. One important reason is that phosphorus stored in the sediments of eutrophied lakes can exchange with the water and thereby keep it nutrient-rich for decades [4]. There are indications that eutrophication has caused many lakes to shift from one state to another. In shallow unstratified lakes, one state is characterised by moderate abundance of phytoplankton, transparent water and vegetated bottoms, the other by high abundance of phytoplankton, turbid water and little or no submerged vegetation. In deep stratified lakes, one state is characterised by an oxygenated hypolimnion and low recycling of phosphorus, and the other by anoxia in the hypolimnion and rapid recycling of phosphorus. Once a lake has reached the latter state, it may tend to remain there even if nutrient concentrations in the water decrease.

The occurrence of 'alternative states' (stable turbid or clear-water states) of pelagic ecosystems can be a consequence of food web interactions [5,6]. Certain food web configurations lead to high abundances of planktivores, or fishes that eat zooplankton. Planktivorous fish species can feed intensively on zooplankton and thereby release phytoplankton from grazing, leading to turbid water. The predation by planktivorous fish can therefore sustain eutrophic conditions in the lake, conditions that are beneficial to the fish themselves, and this feedback may prevent the lake from returning to less eutrophic conditions despite reduced nutrient inputs.

In some cases where eutrophied lakes have failed to recover after a reduction of nutrient supplies, attempts have been made to remedy the problems through intervention in the lakes themselves. Several of the methods tried, including dredging, are very expensive but by no means always successful $[7,8]$.

At least in the short term, however, notable improvements in water quality have been achieved through biomanipulation, usually in the form of decimating the planktivorous fish which typically dominate the fish fauna of eutrophic lakes $[9,10]$. In Eurasia, cyprinids such as roach (Rutilus rutilus) and bream (Abramis brama) are among the most common planktivores in nutrient-rich lakes. In North America, important planktivores of eutrophic lakes include sunfish (Lepomis spp.) and gizzard shad (Dorosoma cepedianum) as well as various cyprinid species.

Reducing the stocks of planktivorous fishes enhances survival of the zooplankton that such fish feed on, and this in turn can reduce the abundance of planktonic algae that serve as food for the zooplankton $[11,12]$. Another reason why removal of planktivorous fish may improve water quality is that the adults of some of these species (e.g. bream and gizzard shad) are also benthivorous. They search for food in the sediments, dispersing nutrient-rich silt and thereby adding to the turbidity and high phosphorus content of the water in eutrophic lakes [13]. Their feeding behaviour may also contribute to the lack of submerged vegetation in such lakes.

The dominance of planktivorous/benthivorous species in eutrophic lakes has been related to the possibility that such species induce an interspecific competitive bottleneck in the recruitment of juvenile predators to predatory (piscivorous) stages, thereby limiting the predation pressure by piscivores [14]. One factor that may induce such a bottleneck is the presence of resources (e.g. cyanobacteria) that are exclusively available to planktivorous/benthivorous species. Another is that many planktivorous/benthivorous species are less affected in their feeding by the low water clarity in eutrophic lakes than visually feeding piscivorous species [14,15].

Ideally, then, a reduction of the populations of planktivorous and benthivorous fish may shift a eutrophied lake back to a less eutrophic state, increasing transparency, allowing benthic vegetation to regain lost ground and decreasing the risk of disturbances such as algal blooms and fish kills. Such changes of lake ecosystem properties - and of the plankton flora in particular may be driven both 'bottom-up' (i.e. by nutrient availability) and 'top-down' (via the upper parts of the food web) [11]. Numerous studies have indicated that aquatic ecosystems may have the potential of being controlled both ways, e.g. [16].

The persistence of biomanipulation effects will partly depend on whether the lake is likely to exhibit alternative stable states or not [17]. For example, this likelihood is greater in shallow lakes and lakes with warm hypolimnia [18]. If alternative states of water clarity do occur, the lake may remain in the new state induced by biomanipulation if it is not destabilised by some other event. If the lake has only a turbid stable state, the rate at which it returns to its previous condition after biomanipulation will among other things depend on the time scale at which the slowest component of its ecosystem operates. 
In most lake food webs, piscivorous fish form the slowest component, with a time scale extending to a decade or more $[19,20]$. This time span is of the same order as that reported for the effects of many biomanipulation attempts.

Removal of planktivores and benthivores for the purpose of lake restoration is usually carried out through intensive fishing, although there are also cases where all fish have been eradicated for this purpose, e.g. through rotenone treatment or temporary emptying of ponds or reservoirs $[21,22]$. An alternative to removing planktivorous and benthivorous fish through direct intervention may be to reduce their dominance by stocking lakes with predatory fish (piscivores) such as pike (Esox lucius). These two approaches have frequently been used in combination - following removal of planktivores and benthivores, piscivores have been stocked in order to prevent zooplankton-feeding fish from regaining their former dominance [23,24]. In some cases, fisheries regulations aiming to increase piscivore biomass have also been used to support biomanipulation (e.g. [25]).

In recent decades, a large number of attempts have been made to restore eutrophic lakes through planktivore decimation or other forms of biomanipulation, not least in Denmark [26], the Netherlands [11] and Finland [27]. Interventions of these kinds have also been the subject of several reviews over the years, e.g. by Søndergaard et al. [7,16], Gulati et al. [8], Meijer et al. [11], Jeppesen et al. [12,28], Hansson et al. [29], Drenner \& Hambright [30] and Hansson [31]. Their approaches and conclusions vary, but in general they have found the likelihood of successful biomanipulation to increase when a) internal and external nutrient loadings have been sufficiently reduced, b) postmanipulation abundance of submerged macrophytes has increased and c) substantial removals have been made of planktivorous fish, and of benthivorous fish in particular. Moreover, fish manipulation by direct removal of planktivorous and benthivorous fish has a higher success rate than stocking of piscivores as a means of controlling planktivores and benthivores [7,8,28,30]. Long-term studies are still not numerous, but they indicate that positive effects of biomanipulation generally last a relatively limited number of years, especially if attempts to reduce internal and external nutrient loadings have failed $[7,8,28]$.

The efficacy of biomanipulation as a means of improving water quality is of considerable interest for lake and water management. In Europe, requirements for measures against eutrophication have become more stringent with the introduction of the EU Water Framework Directive [32]. While such measures mostly involve actions to reduce nutrient loads, biomanipulation has been suggested as an additional or alternative way of achieving 'good ecological status' in eutrophic lakes $[33,34]$. However, the generality of biomanipulation effects on water quality across different lake properties and geographical regions is not known.

\section{Objective of the review}

The purpose of this review is to clarify whether reduction of planktivorous and benthivorous fish may prevent eutrophication problems in lakes. A number of conventional literature reviews on this subject have reported on studies of particular sets of lakes, e.g. providing national overviews of biomanipulation efforts [11,16,27] or analyses based on relatively small international selections of lakes [12,28-30]. Here, instead, we widen the scope - using the 'systematic review' approach [35], we perform a quantitative synthesis of water-quality effects of biomanipulation in temperate eutrophic lakes throughout the world. Rather than reviewing a specific selection or random sample of such interventions, we have have sought to cover all available cases that fulfill our inclusion criteria.

Following an a priori protocol [36], we have thus assembled a large number of studies and screened them for relevance and susceptibility to bias. This has enabled us to extract a substantial amount of quality-assured data on how water quality is affected by biomanipulation. The rigour and transparency of the systematic approach is intended to avoid bias and permit quantitative and repeatable evaluation by means of meta-analysis. Our aim is that this review will provide a useful basis for deciding if and when biomanipulation is useful as a tool for improving water quality in eutrophic lakes.

The review examines full-scale applications of biomanipulation only. While small-scale experimental studies of such interventions can be valuable for clarifying the mechanisms involved, studies of whole-lake manipulation are more relevant when assessing the method as an instrument for environmental management.

In addition to deliberate attempts to improve water quality, we initially also considered unintentional water-quality effects of fish-community changes (caused e.g. by altered fish management practices). Only a few studies of the latter kind of effects were found, however (e.g. [37,38]). Moreover, since unintentional water-quality effects are more likely to have been reported in the scientific literature if they were appreciable than if they were insignificant, inclusion of such studies could increase the risk of publication bias. Therefore, this review covers deliberate biomanipulation efforts only.

\section{Primary question}

What is the influence of a reduction of planktivorous and benthivorous fish (performed directly or indirectly through stocking of piscivores) on water quality in temperate eutrophic lakes?

\section{Components of the primary question}

- Subject (population): Temperate eutrophic lakes anywhere in the world. 
- Intervention: Reduction of populations of planktivorous and benthivorous fish.

This includes removal of planktivorous and/or benthivorous fish, stocking of piscivorous fish and any combination of such interventions. Quantification of the intervention may be based on amounts of fish removed or stocked, and/or on estimates of standing fish stocks before, during and after the intervention.

- Comparator: No intervention.

- Outcomes: Changes of water-quality parameters such as Secchi depth, concentrations of nutrients and chlorophyll $a$ and abundance of phytoplankton. If available, data on changes of communitystructure parameters such as abundance of zooplankton and fish and coverage of submerged macrophytes have also been recorded.

\section{Methods}

\section{Design of the review}

The design of this systematic review was established in detail in an a priori protocol [36]. It follows the guidelines for systematic reviews issued by the Collaboration for Environmental Evidence [39].

As described in the protocol, we developed the review design in close cooperation with stakeholders, primarily in Sweden. Before submission, peer review, revision and final publication of the protocol, a draft version was open for public review at the website of the Mistra Council for Evidence-Based Environmental Management (EviEM) in December 2012 and January 2013. Comments were received from scientists, environmental managers and other stakeholders, and the protocol was revised appropriately.

\section{Searches for literature}

Searches for relevant literature have been made using online publication databases, search engines, specialist websites and bibliographies of literature reviews. Whenever possible, the search strings specified below were applied throughout the searches using online databases, search engines and specialist websites. In several cases, though, they had to be simplified as some sites can handle only a very limited number of search terms or do not allow the use of 'wildcards' or Boolean operators.

Full details of the search strings used and the number of articles found at each stage of the search are provided in Additional file 1.

\section{Search terms}

A scoping exercise had identified the following search terms as being capable of returning a satisfactory set of relevant articles:

- Subject: lake*, reservoir*, pond*, fresh\$water
- Intervention: "manipulat", remov", restor", stock", introduc", reduc*, addition

- Target: "planktivor", "benthivor", cyprinid", piscivor", "predatory fish"", Rutilus, Abramis, Esox, Perca, Stizostedion, Micropterus, Dorosoma, Coregonus, Oncorhynchus, Salmo, roach, bream, pike, muskellunge, perch, pike\$perch, zander, sander, "“"mouth bass", whitefish, cisco, minnow, "gizzard shad".

The terms within each category ('subject', 'intervention' and 'target') were combined using the Boolean operator 'OR'. The three categories were then combined using the Boolean operator 'AND'. An asterisk (") is a wildcard that represents any group of characters, including no character, while a dollar sign (\$) represents zero or one character. The full search string thus reads as follows:

- English: (lake* OR reservoir* OR pond* OR fresh\$water) AND ("manipulat" OR remov" OR restor" OR stock" OR introduc" OR reduc" OR addition) AND ("planktivor" OR "benthivor" OR cyprinid" OR piscivor* OR "predatory fish" OR Rutilus OR Abramis OR Esox OR Perca OR Stizostedion OR Micropterus OR Dorosoma OR Coregonus OR Oncorhynchus OR Salmo OR roach OR bream OR pike OR muskellunge OR perch OR pike\$perch OR zander OR sander OR "“mouth bass" OR whitefish OR cisco OR minnow OR "gizzard shad").

Based on the English search string, the following Danish, Dutch and Swedish search strings were also developed:

- Danish: (sø" OR dam OR mose* OR ferskvand") AND ("manipulat" OR opfisk" OR restau* OR udsæt* OR introduk" OR reduk") AND ("planktivor" OR "benthivor" OR cyprinid" OR piscivor" OR rovfisk" OR fredfisk" OR skidtfisk* OR Rutilus OR Abramis OR Esox OR Perca OR Stizostedion OR Coregonus OR Oncorhynchus OR Salmo OR skalle OR brasen OR gedde OR sandart OR aborre OR *ørred OR helt)

- Dutch: (meer* OR plas* OR zoetwater*) AND (biomanipul" OR "actief biologisch beheer" OR afvissen OR restauratie" OR uitzetten") AND ("planktivor" OR "benthivor" OR planktoneten" OR bodemomwoel" OR piscivor" OR visetende* OR roofvis" OR Rutilus OR Abramis OR Esox OR Perca OR Stizostedion OR brasem OR snoek OR ruisvoorn OR snoekbaars OR karper)

- Swedish: (sjö" OR insjö* OR "magasin" OR "damm* OR sötvatten* OR färskvatten*) AND (biomanipul* OR utfisk" OR reduktionsfisk" OR reducer" OR "restaurer" OR inplanter* OR utplanter" OR utsättning*) AND ("planktivor* OR "planktonäta" OR bent\$ivor* OR bottenäta* OR bottendjursäta* OR cyprinid" OR karpfisk" OR piscivor" OR rovfisk* 


\section{OR Rutilus OR Abramis OR Esox OR Perca OR Stizostedion OR Coregonus OR Oncorhynchus OR Salmo OR mört OR brax* OR gädda OR abborre OR gös OR sik OR *lax OR *öring OR regnbåge).}

No time, language or document type restrictions were applied during the searches.

In addition to searches using the main search string described above, a complementary search was made in a few of the sources mentioned below (Academic Search Premier, Aquatic Sciences and Fisheries Abstracts, Scopus, and Web of Science). The complementary search focused on potential mechanisms and outcomes of biomanipulation, using the following set of search terms:

- Subject: lake*, reservoir*, pond*, fresh\$water

- Target: fish"

- Mechanisms: trophic, cascad", food\$web, top\$down, bottom\$up, resuspen", "stable state*", bistable, "regime shift""

- Outcomes: water\$quality, transparency, clarity, turbid", secchi, "suspended solids", phosph", nitrogen, oxygen, chlorophyll, phytoplankton

\section{Publication databases}

Searches were made in the following online databases:

1). Academic Search Premier

2). Agricola

3). Aquatic Sciences and Fisheries Abstracts

4). Biological Abstracts

5). BioOne

6). COPAC

7). Directory of Open-Access Journals

8). Forskningsdatabasen.dk

9). GeoBase

10). IngentaConnect

11). JSTOR

12). Libris

13). PiCarta

14). Scopus

15). SpringerLink

16). SwePub

17). Web of Science

18). Wiley Online Library.

\section{Search engines}

Internet searches were also performed using the following search engines:

Google (www.google.com)

Google Scholar (scholar.google.com)

Growyn

Scirus.
In each case, the first 100 hits (based on relevance) were examined for appropriate data. Potentially useful documents that had not already been found in publication databases were recorded.

\section{Specialist websites}

Websites of the specialist organisations listed below were searched for links or references to relevant publications and data, including 'grey literature'. Potentially useful documents that had not already been found using publication databases or search engines were recorded.

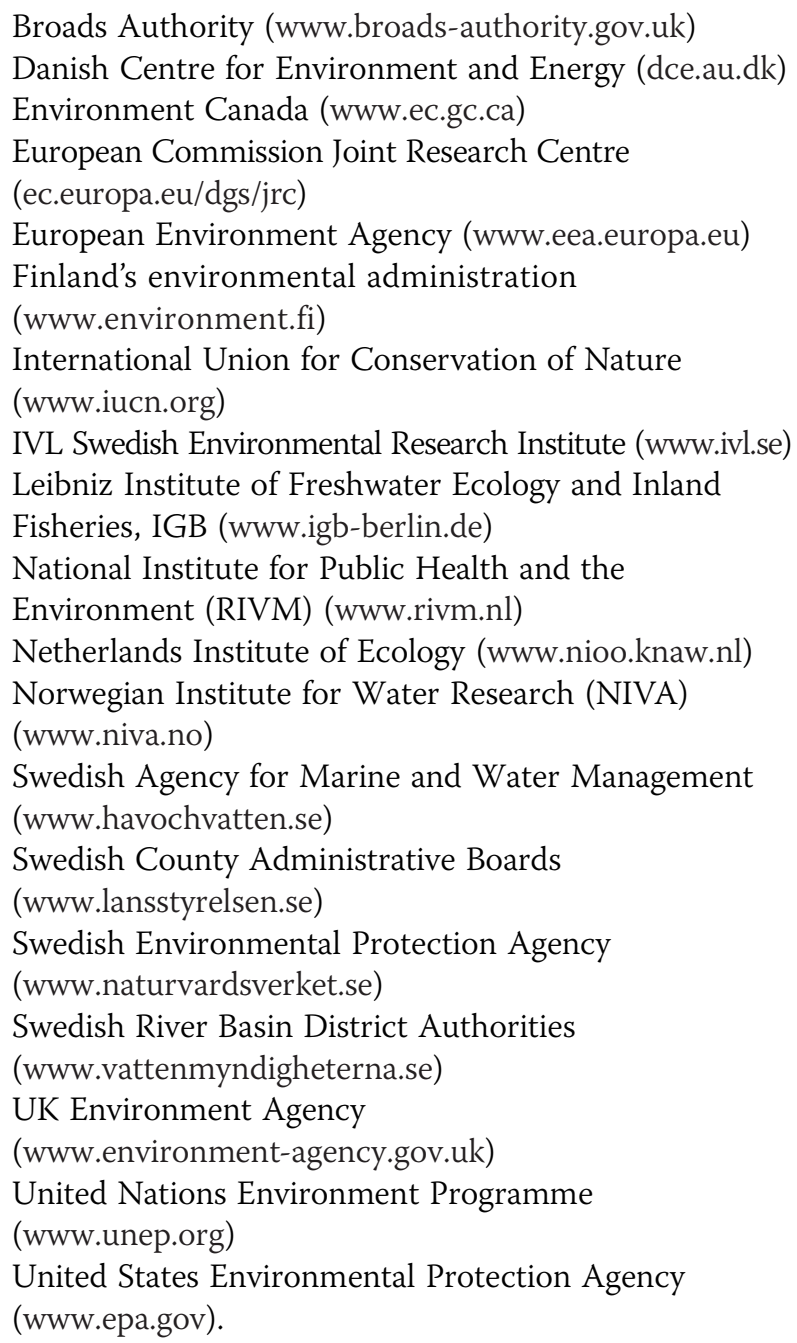

\section{Other literature searches}

Relevant literature was also searched for in bibliographies of literature reviews such as those mentioned in the Background section. Potentially useful documents that had not already been found in online sources were recorded. A few more articles were brought to our attention by stakeholders.

In addition, unpublished data were in some cases made available by e.g. study authors, consultants or 
local authorities involved in biomanipulation projects. Stakeholders had been asked to suggest suitable contacts.

\section{Search update}

An update to the literature searches was made in late 2013, about ten months after the main searches. The update involved searches in Web of Science and Google Scholar using the main English search string. Web of Science was also searched with the complementary search string.

\section{Screening Screening process}

Articles found by searches in databases were evaluated for inclusion at three successive levels. First they were assessed by title by a single reviewer (CB). In cases of uncertainty, the reviewer chose inclusion rather than exclusion. As a check of consistency, a subset of 100 articles was assessed by all members of the review team. Since this check showed that the main reviewer was considerably more inclusive than the average team member, it seemed safe to proceed with the screening without modification or further specification of the inclusion/exclusion criteria.

Next, each article found to be potentially relevant on the basis of title was judged for inclusion on the basis of abstract, again by a single reviewer $(\mathrm{CB})$ who in cases of uncertainty tended towards inclusion. A second reviewer (LP) assessed a subset consisting of 199 (10\%) of the abstracts, and the agreement between the two reviewers' assessments was checked with a kappa test. Since the outcome, $\mathrm{\kappa}=0.71$, indicated a 'substantial' agreement [40] and since the inconsistency had chiefly been caused by the main reviewer being more inclusive than the second one, the screening was allowed to proceed without revision.

Finally, each article found to be relevant on the basis of abstract was judged for inclusion by a reviewer studying the full text. This task was shared by all members of the review team. The articles were randomly distributed within the team, but some redistribution was then made to avoid having reviewers assess studies authored by themselves or articles written in an unfamiliar language. Articles found using search engines, specialist websites, review bibliographies or stakeholder contacts were also entered at this stage in the screening process. Doubtful cases - articles that the reviewer could not include or exclude with certainty even after having read the full text - were discussed and decided on by the entire team.

A list of all articles rejected on the basis of full-text assessment is provided in Additional file 2: Table B together with the reasons for exclusion. This file also contains a list of potentially relevant articles that were not found in full text (Additional file 2: Table A).

\section{Study inclusion criteria}

Each study had to pass each of the following criteria in order to be included, either by providing all the required data itself or by referring to other articles where supplementary information was presented.

- Relevant subjects: Temperate freshwater lakes or reservoirs (with an area equal to or larger than 1 hectare) characterised by study authors as eutrophic (or hypertrophic) and/or having summer concentrations of total phosphorus (TP) exceeding $30 \mu \mathrm{g} / \mathrm{l}$ before biomanipulation.

- Relevant types of intervention: Removal of planktivorous or benthivorous fish, stocking of piscivorous fish and any combination of such interventions, provided that the intention was to improve water quality.

- Relevant type of comparator: No intervention.

- Relevant types of outcome: Change of Secchi depth, change of concentrations of chlorophyll $a$, total phosphorus, total nitrogen, oxygen or suspended solids, or change of total phytoplankton or cyanobacteria abundance.

- Relevant types of study: Any primary field study of water quality in lakes or reservoirs (or in artificially separated compartments with areas $\geq 1$ ha in such water bodies) that had been subject to large-scale biomanipulation of any of the kinds described above. The study could be based on before/after comparisons or site comparisons or both (see Study quality assessment below).

During screening on full text, the following inclusion criterion was also applied:

- Language: Full text written in English, Danish, Dutch, German, Norwegian or Swedish.

\section{Potential effect modifiers and reasons for heterogeneity}

To the extent that data were available, the potential effect modifiers listed below were considered and recorded. This was done on a lake-by-lake rather than article-by-article basis.

\section{Geographical coordinates}

Altitude

Lake area

Mean and maximum lake depth

Retention time

Lake connectivity (whether the lake had tributaries and/or connections to other lakes)

Lake salinity

Water colour

Concentration of dissolved organic carbon (DOC) 
Occurrence of stratification in the lake

Annual mean temperature

Presence of introduced species

Presence of grazing or piscivorous birds

Study duration and seasonality

History of biomanipulation (years and seasonality of interventions, amounts of fish removed or stocked, methods for fish removal, species, age and size of stocked fish, etc.).

History of other interventions and disturbances, e.g.

1) other in-lake attempts to mitigate eutrophication problems (such as dredging, aeration, improvement of recruitment habitats for predatory fish etc.);

2) external supplies of phosphorus (and other pollutants) from point sources and runoff, internal nutrient loading and any experimental nutrient additions to the lake;

3) land use in the surrounding area (including attempts to reduce nutrient losses by modifying the use of fertilisers, establishing buffer zones with permanent vegetation between fields and watercourses etc.);

4) damming, lake lowering and other hydrological disturbances;

5) special weather conditions (droughts, heat waves, storms);

6) fisheries and stocking not intended as a means of biomanipulation;

7) natural or unintended anthropogenic fish-kills.

\section{Study quality assessment}

In many cases, the biomanipulation of an individual lake has been described in several articles that cover different aspects of the intervention and its consequences. One article may focus on the stocking or removals of fish and how they have affected standing fish stocks, whereas details on how this intervention has influenced water quality may be found elsewhere.

For this reason, once the full-text screening of articles was completed, the review proceeded on a lake-by-lake rather than article-by-article basis - all articles with relevant data on a certain lake or biomanipulation project were considered together. Contrary to what was stated in the protocol [36], therefore, quality assessment of studies that had passed full-text screening was based on the entire evidence found on a certain lake biomanipulation, not on individual articles. A few articles that initially had been excluded due to absence of relevant water-quality data were re-entered at this stage, since they contained useful data on other aspects of a biomanipulation project.

The quality assessment was performed by the six ecologists in the review team (SRC, AG, PL, LP, CS and EVD) - again with care taken that reviewers would not assess articles authored by themselves - and double- checked by the seventh member of the team (CB). Doubtful cases were discussed and decided on by the entire team.

\section{Exclusion criteria}

If the combined evidence on a biomanipulated lake had any of the deficiencies listed below, it was considered to have high susceptibility to bias. In such cases, the lake was excluded from the review.

- No (or insufficient) data on water quality before biomanipulation. The available data were regarded as insufficient if they covered less than one full pre-manipulation summer season.

- No useful quantitative data on fish removals or changes of standing fish stocks.

- Insufficient methodological description.

A list of lakes rejected on the basis of quality assessment is provided in Additional file 3 together with the reasons for exclusion.

\section{Additional quality criteria}

For lakes that were not rejected based on the above exclusion criteria, the combined evidence was considered to have either low or medium susceptibility to bias. If any of the criteria listed below applied, susceptibility to bias was classified as medium. If none of them applied, susceptibility to bias was considered to be low (meaning that the quality of evidence was regarded to be high).

- Confounding interventions or disturbances. Interventions like aeration, dredging, aluminium treatment or sewage diversion (or disturbances like fish-kills) occurred just before, during or just after fish manipulation.

- Insufficient data on potential effect modifiers. Available lake metadata and data on lake history were so incomplete that they allowed no conclusions on whether other interventions or disturbances had occurred besides fish manipulation.

- No useful data on within-year water-quality variation. Available water-quality data consisted of only one observation per year or of annual means without standard deviations, standard errors, confidence intervals or similar measures of variation.

- Multiple basins. The lake or lake system consisted of at least two basins that were manipulated differently and/or had markedly different water quality.

\section{Data extraction strategy}

Annual means and variation of summer-season waterquality data have been extracted from tables and graphs in articles and reports, using image analysis software 
(WebPlotDigitizer) when necessary. In some cases, study authors or database managers were asked to supply data in digital format. This was done where useful data had been published in graphs from which they were difficult to extract accurately enough, or when it was known or assumed that considerable amounts of relevant but unpublished data could be available in addition to the published results.

In cases where raw data were received, summary statistics have been calculated by us. Where individual waterquality data have been available, multi-year means and variation have been calculated based on these data rather than on annual averages.

The summer season has been defined differently by different authors, but 1 May - 30 September is the most common choice. This was also the period that we used ourselves when selecting relevant raw data (although our search for data was global, all biomanipulations found suitable for quantitative analysis had been performed in the northern hemisphere).

Data on potential effect modifiers and other metadata were extracted from the included articles whenever available, but data on annual means of the atmospheric temperature were downloaded from the WorldClim database [41].

Initially, outcomes and metadata were recorded in a separate Excel file for each included lake. Data to be used in meta-analysis were then transferred to an Access database.

\section{Definitions of pre-, during- and post-manipulation periods}

Most studies of biomanipulations have a Before/After ('BA') design - they compare data that have been collected prior to and following the intervention (or at least during different stages of the intervention). Since a biomanipulation may extend over several months or even years, BA studies often present data sampled not only before and after but also during the intervention. Due to the complexity of many biomanipulation projects, however, it is not always obvious when the main intervention started or ended. For instance, mass removals of fish may have been preceded or followed by less significant fish removals, and stocking may have taken place not only after periods of mass removal but also before or during them.

For intervention involving fish removal, we defined the main biomanipulation period as the years during which significant amounts of fish (at least 7-8 kg per hectare) were removed. Piscivore stocking performed within this period was normally seen as part of the main biomanipulation, but not if the fish removal resulted in complete eradication of the fish stocks. For interventions based on stocking only, the main biomanipulation period was defined as the years during which adult piscivores or significant numbers of young piscivores (at least 50-100 individuals per hectare) were stocked. A single year with insignificant or no fish removal or stocking was included in the main biomanipulation period if it was both preceded and followed by years with significant manipulation.

Building on these definitions, we applied the following rules to decide whether water-quality data sampled during a certain summer season represented Before, During or After conditions in the manipulated lake. Data that could not be included in any of these categories were not used.

The Before period was defined to stretch back as long as water-quality data were available and pre-manipulation summer conditions (concentrations of total phosphorus and chlorophyll $a$, Secchi depth etc.) were reasonably stable. If confounding interventions or disturbances (e.g. aeration, dredging, in-lake chemical treatment, significant increases or decreases of phosphorus inputs, or fish-kills due to oxygen deficiency) took place during the premanipulation period, the Before period was said to start after the last onset or end of such events. The Before period was defined to end with (and include) the last premanipulation summer. Periods without water-quality data were included in the Before period if they lasted no more than 5 years and were preceded by a year with waterquality data.

The During period was defined to begin with the first during- or post-manipulation summer and conclude with the last year with significant biomanipulation. This means that no summer season was categorised as 'During' if the manipulation was confined to a single autumn.

The After period was defined to begin with the first post-manipulation year and last as long as water-quality data were available and no additional interventions or confounding events began. Periods without water-quality data were included in the After period if they lasted no more than 5 years and were followed by a year with water-quality data.

Two biomanipulations of a single lake were regarded as distinct interventions (to be analysed individually) if they were separated by at least $8-10$ years without significant manipulation. The last 3 years before the second biomanipulation were then defined as the Before period of that intervention.

\section{Data synthesis and presentation}

Meta-analysability and selection of a high-quality dataset

Although we have access to water-quality data for each of the biomanipulation projects included in this review, a considerable part of these projects do not appear in any of the meta-analyses described below. One reason is that for some biomanipulations, the available data do not include any of the water-quality parameters covered by the metaanalyses (Secchi depth, chlorophyll $a$ concentration and cyanobacteria abundance). Another reason is that some of the data available to us are not meta-analysable due to 
absence of useful information on variation (such as standard deviations, standard errors or confidence intervals) or on the number of observations. Published data on water quality in manipulated lakes sometimes consist of single measurements per year or of summer averages without any information on within-year variation. In other cases, published summer means or medians are accompanied by fractiles or ranges, but there is no reliable way of converting such data to measures of variation that can be used in meta-analyses.

Where water-quality data were available for more than one year within a Before-, During- or After-manipulation period, calculation of interannual variation enabled us to include them in some meta-analyses even if there was no useful information on within-year variation. However, due to the large seasonal fluctuations of primary production and phytoplankton abundance that characterise most eutrophic lakes, within-year variation of water quality may be larger than the interannual variation, even if the analysis is restricted to data sampled during summer. If this is the case, we may introduce bias by using effect sizes with interannual variation only, since such data will then tend to have lower variance and hence be given higher weight in meta-analyses than if their within-year variation had been known and included too.

Another important quality aspect is the presence or absence of confounding interventions or disturbances. Biomanipulation has frequently been performed in combination with other efforts to improve water quality, such as aeration or artificial mixing of deep waters, dredging (sediment removal), sewage diversion or other reductions of external nutrient inputs, or in-lake phosphorus removal with aluminium or iron salts. In many eutrophic or hypertrophic lakes, moreover, fish-kills caused by oxygen deficiency may have water-quality effects resembling those of deliberate manipulations of the fish fauna.

For these reasons, much of our analysis uses a highquality 'selected dataset' where effect sizes based on single data per year and/or confounded data have been excluded. An alternative way of identifying a high-quality dataset would have been to include effect sizes only for biomanipulations where data were categorised as having low susceptibility to bias. The classification of susceptibility to bias is somewhat coarse, however, being based on the combined evidence on a biomanipulation project rather than on individual effect sizes. Even for the same biomanipulation, some effect sizes may be based on confounded data or single data per year, while others are not.

\section{Meta-analyses}

The impacts of biomanipulation on water quality were mainly analysed using meta-analytical approaches. The meta-analyses were carried out using the metafor package [42] within the R environment v. 3.0.2 [43].
Most of the meta-analyses used water transparency (measured as Secchi depth) or chlorophyll $a$ concentration as response variables. Since all data for these variables could be converted to the same units ( $\mathrm{m}$ and $\mu \mathrm{g} / \mathrm{l}$, respectively), the comparisons were based on mean differences. The effect sizes were calculated as the difference between the mean response during or after the main biomanipulation period and the mean response before the manipulation. Positive effect sizes thus indicate that the response parameter was higher during or after intervention than before intervention. When analysing effect sizes based on the selected dataset, we also explored the consequences of exchanging mean differences for mean log ratios.

Moreover, a few meta-analyses were made of data on cyanobacteria abundance. Since these data were given in several incommensurable units, mean log ratios were used as effect sizes for the cyanobacteria meta-analyses.

Random effects models were developed for each response variable, comparing data acquired Before/During or Before/After manipulation. For the Before/After comparisons, models were developed for each of the first 7 years after manipulation, as well as the average of years 1-3 after manipulation. Random effects models were run using restricted maximum likelihood to estimate heterogeneity, and data are presented in forest plots showing mean effect sizes and 95\% confidence intervals. Random effect models were also developed for separate subgroups of comparisons, covering various aspects of data quality and different types of biomanipulation.

To investigate to what extent lake properties and biomanipulation methods influence the effects of biomanipulation on Secchi depth and chlorophyll $a$ concentrations, we performed meta-regressions on Before/During and Before/After comparisons (the latter covering years 1-3 after manipulation). The most relevant effect modifiers - lake area, mean depth, retention time, pre-manipulation total phosphorus (TP) concentration, mean annual atmospheric temperature, duration of fish removals, amount of fish removed (expressed as $\mathrm{kg} / \mathrm{ha}$ or $\mathrm{kg} / \mathrm{ha} / \mathrm{yr}$ ) and depletion of fish stocks - were used as co-variates.

Data were not plentiful enough to allow a complete analysis using all explanatory variables simultaneously. However, since lake area, mean depth and pre-manipulation TP concentration were highly correlated (see Additional file 4), we applied principal component analysis (PCA) to convert observations of these lake properties into a set of linearly uncorrelated variables (principal components, $\mathrm{PC})$. We then used the first PC (PC1) as an explanatory variable in the meta-regressions.

$\mathrm{PC} 1$ explained $80 \%$ or more of the variation in the three selected lake properties, reflecting increasing lake area and decreasing pre-manipulation TP concentrations, whereas mean depth was mainly reflected in PC2 that only explained a minor part of the variation (see Additional file 5). 


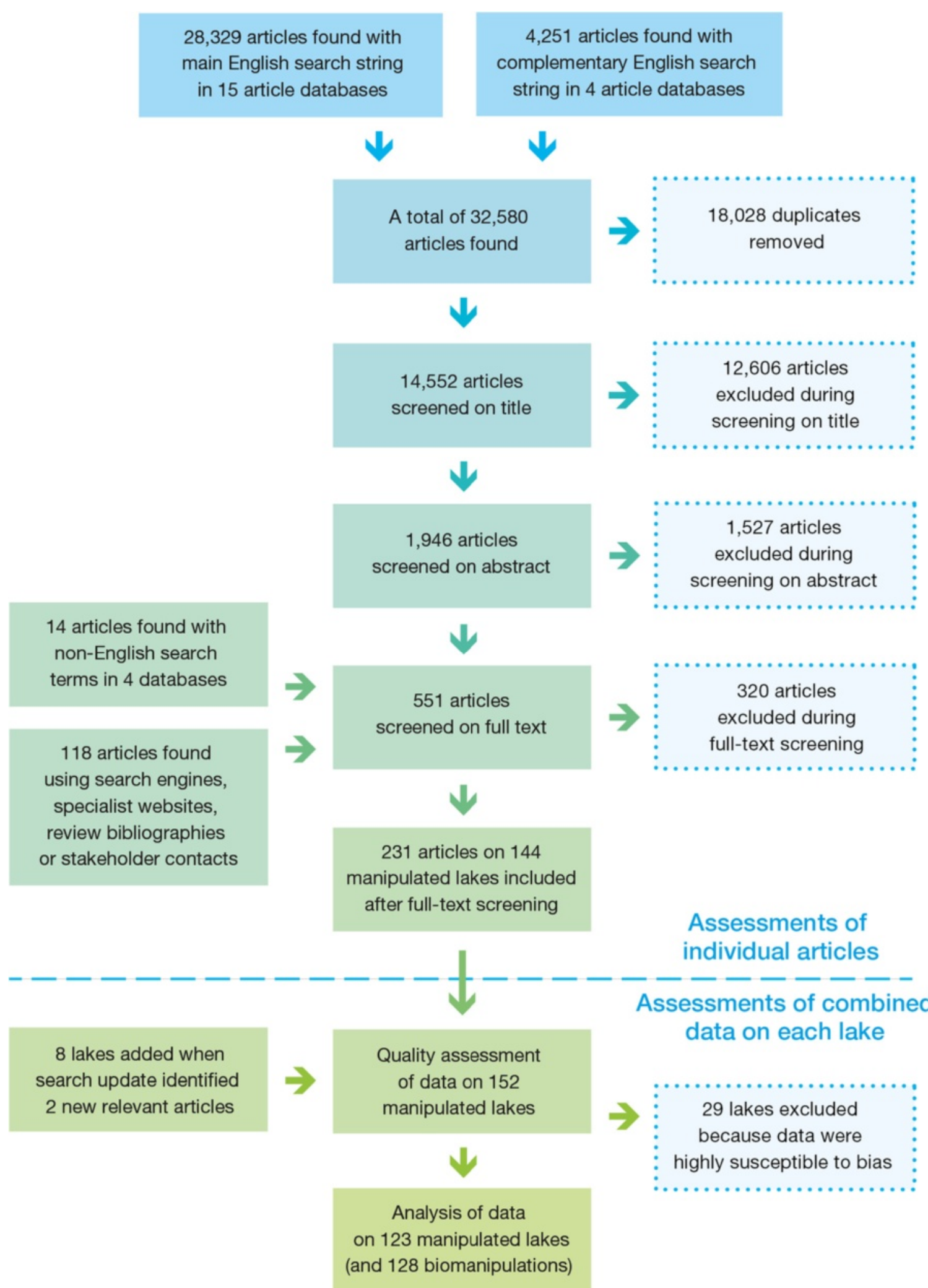

Figure 1 Overview of article inclusion, article screening and quality assessment of lake data. 
Table 1 Susceptibility to bias of the evidence on included biomanipulations

\begin{tabular}{ll}
\hline & No. of cases \\
\hline Low & 53 \\
$\begin{array}{l}\text { Medium due to confounding interventions or } \\
\text { disturbances }\end{array}$ & 31 \\
$\begin{array}{l}\text { Medium due to insufficient data on potential effect } \\
\text { modifiers }\end{array}$ & 13 \\
$\begin{array}{l}\text { Medium due to absence of useful data on within-year } \\
\text { water-quality variation }\end{array}$ & 43 \\
$\begin{array}{l}\text { Medium since the lake consisted of multiple basins with } \\
\text { different interventions or water quality }\end{array}$ & 6 \\
\hline
\end{tabular}

The evidence on some biomanipulations has medium susceptibility to bias based on more than one of the quality criteria.w

Meta-regression models were made using the combined 'lake-property' variable (PC1), a measure of intervention strength (fish removals expressed as $\mathrm{kg} / \mathrm{ha} / \mathrm{yr}$ ), and the interaction between these two as explanatory variables. Selection between the models (including the intercept-only model) was based upon minimum Akaike's information criterion corrected for small sample size (AICc).

Since we were not able to test all effect modifiers listed above at the same time, we also performed metaregressions with each of them separately.

All meta-regressions were based on the selected dataset, with stocking-only biomanipulations excluded (see Results). Due to skewness of the data, lake areas, mean depths, retention times, pre-manipulation TP concentrations and amounts of fish removed were log-transformed before analysis.

Finally, Secchi depth and chlorophyll $a$ data (both from the selected set and from the entire set of meta-analysable data) were tested for possible publication bias using funnel plots.

\section{Results}

Review descriptive statistics

Literature searches and screening

The main searches for literature were conducted between 10 December 2012 and 4 March 2013, and an update was made on 26 October 2013.
Searches with the main English search terms in 15 publication databases returned a total of 28,329 articles (or 12,908 after removal of duplicates) - see Figure 1. Four of the databases (Academic Search Premier, Aquatic Sciences and Fisheries Abstracts, Scopus, and Web of Science) were also searched with the complementary search string, which returned a total of 4,251 articles (or 2,270 after removal of duplicates). Of these articles, 1,644 had not been found with the main search string.

After title screening of the 14,552 unique publications found by the main and complementary searches, 1,946 of them remained included. Screening based on abstract left 419 articles that still were considered as potentially relevant. Most of the excluded articles contained no relevant information on how water quality had responded to biomanipulation, or did not touch on reductions of planktivorous or benthivorous fish at all (see Additional file 6).

Searches with Danish, Dutch and Swedish search terms in national bibliographic databases yielded 4,3 and 7 potentially relevant publications in these languages, respectively. Searches using search engines returned 33 potentially relevant articles (17 found with English search terms, 10 with Danish and 6 with Swedish ones) in addition to those that already had been identified. Similarly, searches on specialist websites located another 9 potentially useful publications (2 found using English search terms and 7 using Danish ones). An additional 38 articles were found in bibliographies of literature reviews, while 38 more were added by members of the review team or included as a result of stakeholder contacts or Google searches for the names of known biomanipulated lakes. A large part of the publications referred to in this paragraph can be characterised as grey literature.

In all, the searches resulted in 551 articles to be screened based on full text. After screening, 231 of them were still included. At this stage, the most common reason for exclusion was that studies contained no relevant primary data (see Additional file 6 and Additional file 2: Table B). In 22 cases, publications had to be excluded because they were not found in full text (see Additional file 2: Table A). When the search for publications was updated in late 2013, two new articles were included.

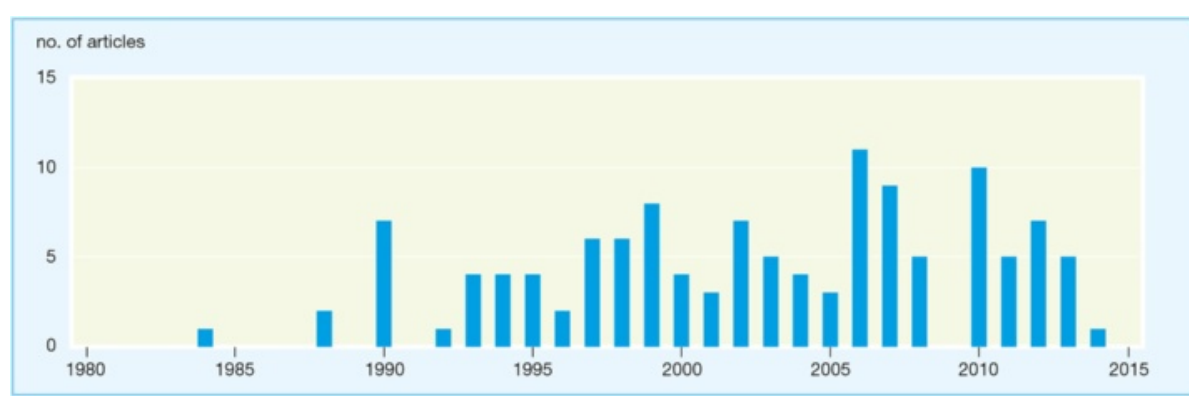

Figure 2 Year of publication of the 124 articles that were used for data extraction. 


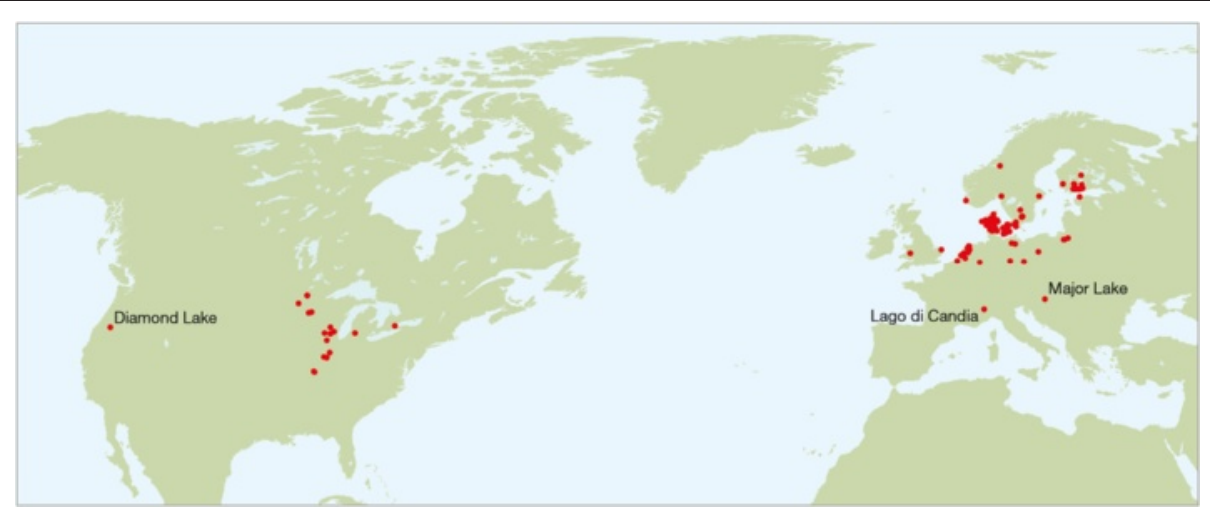

Figure 3 Locations of biomanipulated lakes included in the review. More detailed maps are available in Additional file 9

\section{Quality assessment}

The 233 articles that had passed full-text screening described a total of 152 biomanipulated lakes. A single lake could be referred to in up to twenty different articles, while a single publication could describe a large number of different manipulation projects. Quality assessment of the available evidence was therefore performed per lake rather than per article.

This assessment led to the exclusion of 29 lakes from the review, since the evidence found on them was categorised as highly susceptible to bias. The most common reason for exclusion was that data on pre-manipulation water quality were insufficient or entirely absent (see Additional file 3).

In 5 of the 123 manipulated lakes that remained included in the review, interventions had been performed twice at sufficiently long intervals (8-10 years or more) that they could be regarded as independent of each other. Therefore, 128 individual biomanipulations have been considered in this review.

For 53 of the 128 biomanipulations we found the quality of the available evidence sufficient to have low susceptibility to bias. In the remaining 75 cases, we classified the susceptibility to bias as medium (see Table 1 and Additional file 7: Table B).

\section{Sources of articles used for data extraction}

Although 233 articles had been judged as relevant during full-text screening, only 124 of them were actually used for extraction of data. In some cases, the reason for not using an article was that it related to a lake that had been excluded during quality assessment, but the most common reason was that articles were redundant for the purposes of this review - they reported data that could also be found elsewhere (see Additional file 2: Table C and D). Many of them were reviews rather than sources of primary data.

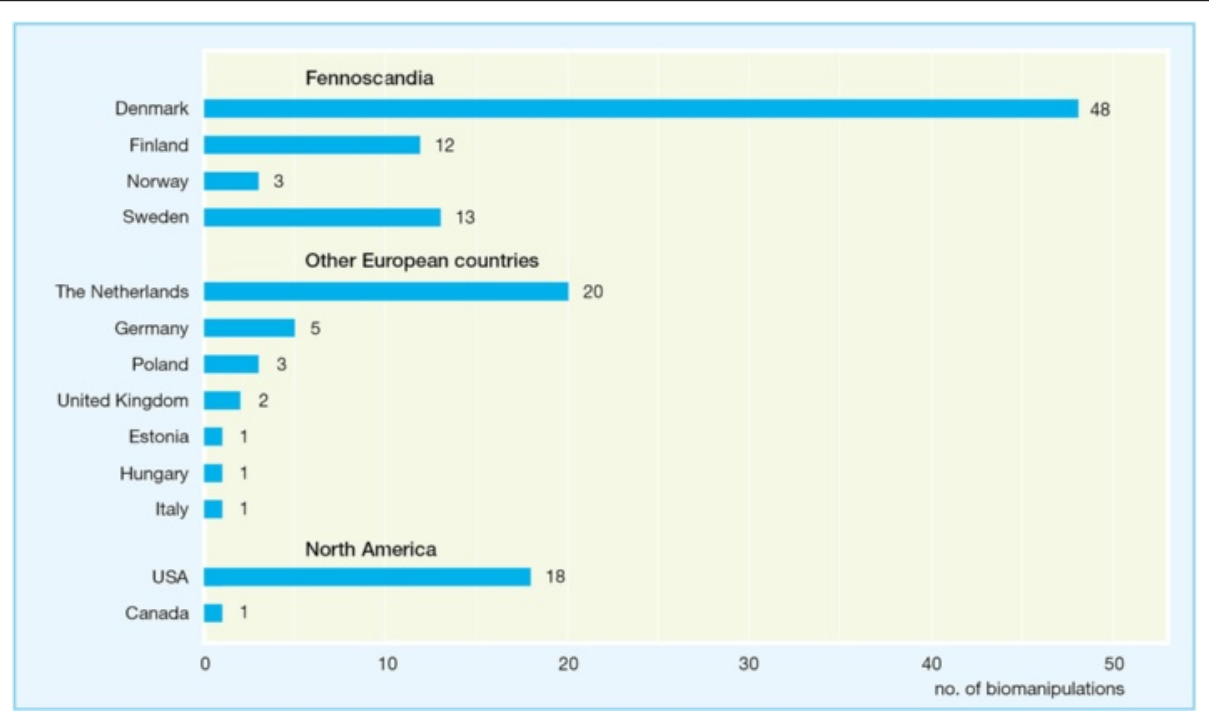

Figure 4 Number of included biomanipulations per country. 
Table 2 Characteristics of included lakes

\begin{tabular}{llll}
\hline & Median & Min. & Max. \\
\hline Mean depth $(\mathrm{m})$ & 2.1 & 0.7 & 13.5 \\
Lake area (ha) & 37 & 1.2 & 3985 \\
Retention time (days) & 220 & 1 & 3870 \\
$\begin{array}{l}\text { Total phosphorus concentration } \\
\text { (pre-manipulation summer mean, } \mu \mathrm{g} / \mathrm{l})\end{array}$ & 133 & 25 & 1195 \\
Mean annual atmospheric temperature $\left({ }^{\circ} \mathrm{C}\right)$ & 7.8 & 1.3 & 13.1 \\
\hline
\end{tabular}

Of the 124 articles that were used for data extraction, 69 had been found in publication databases (see Additional file 8). Of these, 61 were identified using the main English search terms, while 5 others were found with the supplementary search string only and 3 with Dutch or Swedish search terms.

Of the remaining 55 articles used for data extraction, we had found 35 using search engines (mostly by searching for names of known biomanipulated lakes), 4 at specialist websites, 5 in review bibliographies and 3 through stakeholder contacts, whereas 8 had been provided by members of the review team. While 77 articles were written in English, 30 were in Danish, 3 in Dutch, 2 in German and 12 in Swedish.

Only 3 of the 124 articles were published before 1990 . Years of publication of the more recent articles were distributed fairly evenly over the period 1990-2013 (see Figure 2).

\section{Narrative synthesis \\ Overall characteristics of included lakes and biomanipulations}

Most of the biomanipulations covered by this review were carried out in central or northern Europe - more than half of them in Fennoscandia alone - whereas the remaining 15\% were performed in North America (see Figures 3 and 4 and Additional file 9). Our literature searches also identified a number of biomanipulated lakes in temperate parts of Asia, Australia and South America, but all of these cases were excluded during full-text screening or quality assessment.
The included lakes are typically shallow, small, and hypertrophic rather than merely eutrophic (see Table 2). Based on the available literature, 73 of them were categorised as natural lakes (although some of these have been lowered or modified in other ways), while 8 were characterised by study authors as artificial lakes, 11 as impoundments and 16 as former peat, sand or gravel pits (see Additional file 7: Table A).

Of the 128 individual lake biomanipulations in the review, 102 included fish removal. In 81 of these cases, stocks of planktivorous and/or benthivorous fish were decimated solely by fishing. Eleven other manipulations involved rotenone or other piscicides, while ten included partial or complete emptying of the lake or reservoir, often but not always in combination with fishing (see Additional file 7: Table C). Several of the latter interventions resulted in complete eradication of all fish species. In 35 cases where planktivorous and benthivorous fish were decimated, this intervention was combined with stocking of piscivores such as northern pike (Esox lucius), pikeperch (Sander lucioperca) or perch (Perca fluviatilis). The biomanipulations reviewed by us also include 26 cases solely based on piscivore stocking.

Details on the included biomanipulations are presented in three tables in Additional file 7. Table A in this file provides basic data on the manipulated lakes: location, lake type, lake area, mean depth, occurrence of stratification in summer, retention time, average pre-manipulation concentration of total phosphorus in summer, and mean annual atmospheric temperature. Table B presents study design, assessments of study quality, basic data on the main biomanipulation (type, timing and duration), and a selection of water-quality data (summer averages of Secchi depth and chlorophyll $a$ concentration before and during the main biomanipulation and in the first three postmanipulation years). Table $\mathrm{C}$ provides details about fish removals and/or fish stockings included in the main biomanipulation, and also available data on changes of standing fish stocks.

Table 3 No. of biomanipulations with available effect sizes

\begin{tabular}{|c|c|c|c|c|c|c|}
\hline & \multicolumn{3}{|c|}{ Before/During effect sizes } & \multicolumn{3}{|c|}{ Before/After effect sizes* } \\
\hline & All & Meta-analysable & Selected dataset & All & Meta-analysable & Selected dataset \\
\hline Chlorophyll a concentration & 87 & 75 & 30 & 73 & 65 & 26 \\
\hline Secchi depth & 94 & 81 & 34 & 78 & 66 & 27 \\
\hline Total phosphorus concentration & 106 & 81 & 28 & 92 & 71 & 27 \\
\hline Cyanobacteria abundance & 35 & 27 & 13 & 23 & 13 & 5 \\
\hline Total phytoplankton abundance & 39 & 29 & 13 & 24 & 13 & 4 \\
\hline Daphnia abundance & 22 & 15 & 8 & 22 & 12 & 6 \\
\hline Cladocera abundance & 24 & 15 & 8 & 23 & 13 & 7 \\
\hline Total zooplankton abundance & 23 & 14 & 8 & 20 & 10 & 6 \\
\hline
\end{tabular}

*Data available for at least one of the first three post-manipulation years. 
Availability of water-quality data and other outcomes

The availability of water-quality data from different stages of each of the included biomanipulation projects is shown in Figures 5 and 6. This figure also indicates where available data have not been used due to confounding interventions or disturbances.

Of the 128 biomanipulations included in the review, 125 are covered by studies that - in a wide sense - have a 'BA'

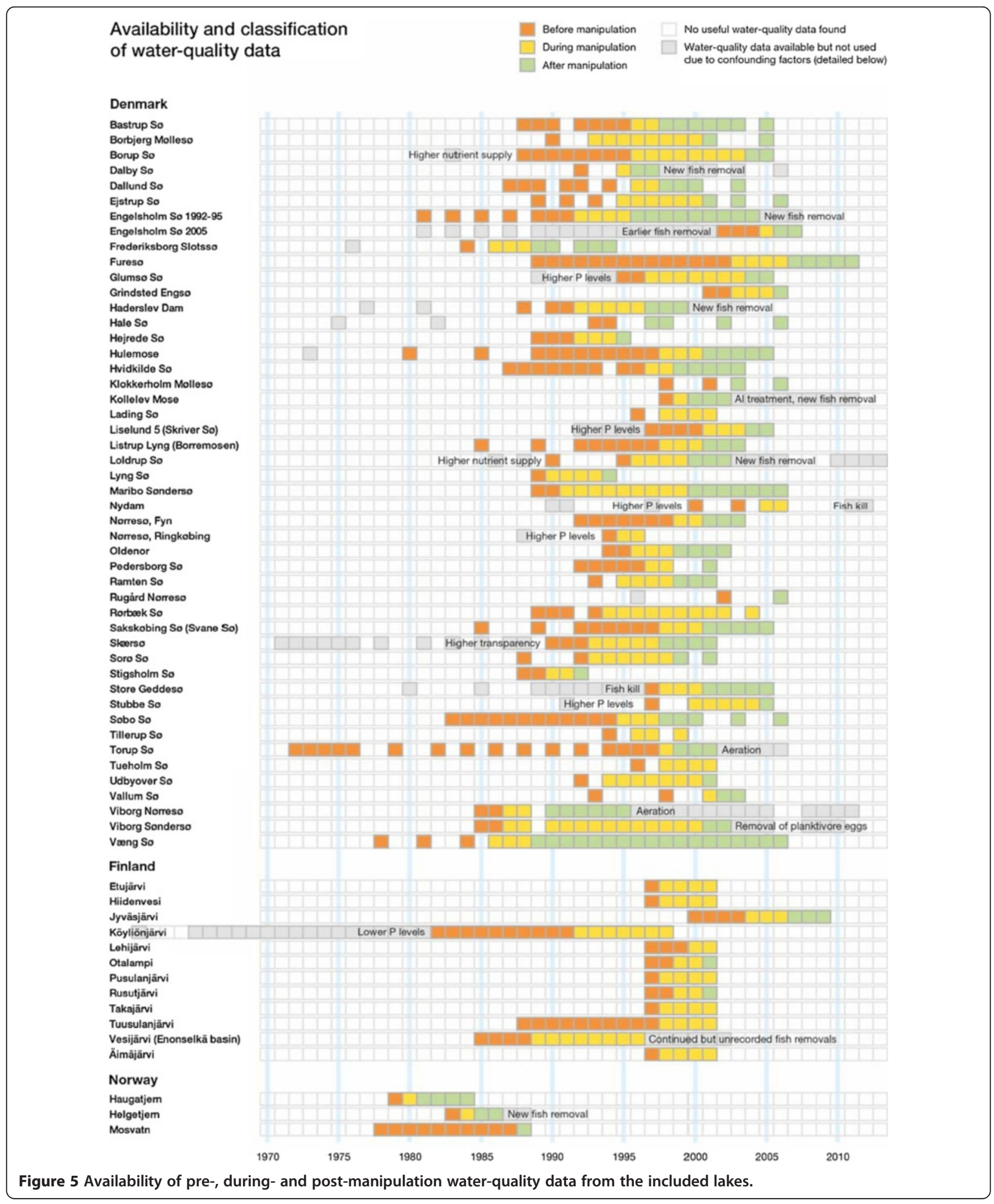


(Before/After) design. In 86 of these cases, we have access to water-quality data sampled not only before and after but also during the main biomanipulation, and we therefore refer to them as having a 'BDA' (Before/During/After) design (see the Methods section). In 27 other cases, we have data collected before and during the biomanipulation, but not afterwards. We refer to such cases as having a 'BD' (Before/During) design. The remaining 12 cases

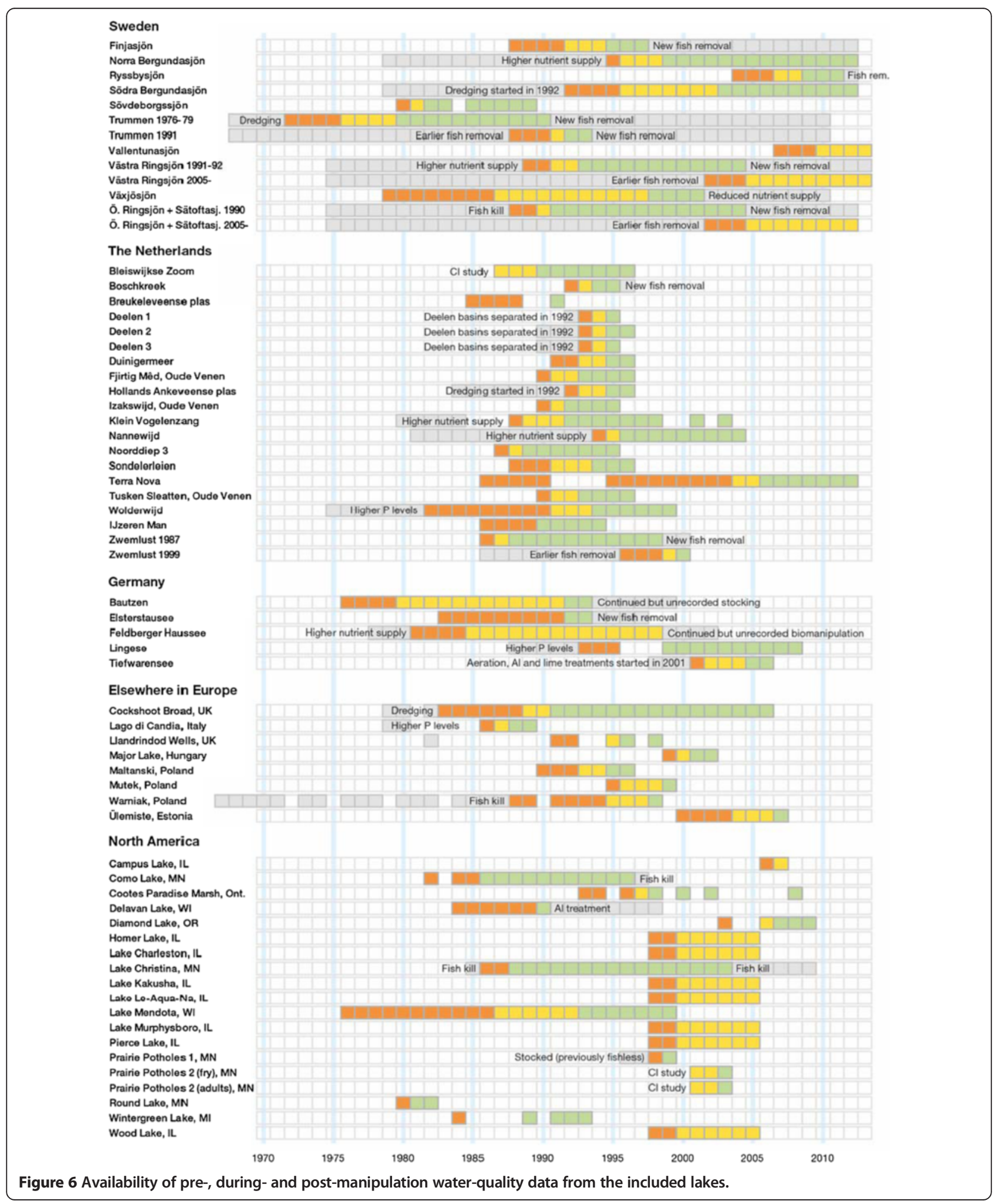


may be called 'true BA', since in these cases we have access to data collected before and after but not during the manipulation.

Three of the biomanipulations in the review - Bleiswijkse Zoom, Prairie Potholes 2 (adults) and Prairie Potholes 2 (fry) - are covered by studies that present no premanipulation data. Instead, these studies are based on comparisons between the manipulated lakes and similar lakes where no such intervention has taken place. This means that they have a 'CI' (Comparator/Intervention) design. In our quantitative synthesis, CI comparisons made during biomanipulation are included among Before/During comparisons, whereas $\mathrm{CI}$ comparisons made after biomanipulation are included among Before/After comparisons.

The outcomes that we have extracted from articles and databases are dominated by observations of Secchi depth, chlorophyll $a$ and total phosphorus. We also extracted data on abundances of cyanobacteria, total phytoplankton, Daphnia, Cladocera and total zooplankton, although such information was found for relatively few of the biomanipulations (see Table 3). Data on oxygen levels, concentrations of suspended solids and cover of macrophytes were found to be too scarce and/or heterogeneous to be useful. We have also chosen not to use data on total nitrogen concentrations - such data are frequently available in the literature, but they have limited relevance to lake eutrophication.

\section{An overview of all available Secchi depth and chlorophyll a data}

The biomanipulations reviewed here include interventions of highly varying strength, ranging from very modest planktivore/benthivore removal (only $13-30 \mathrm{~kg} / \mathrm{ha} / \mathrm{yr}$ in some cases) or stocking of limited numbers of piscivores to complete eradication of the entire fish fauna. Moreover, they have been performed in a set of lakes that covers wide ranges of size, depth, trophic status and climatic conditions.

Yet, even a cursory inspection of the outcomes indicates that a clear majority of the interventions have had positive effects on water quality (see Figure 7 and Additional file 7: Table B). Secchi depths have in most cases increased, whereas concentrations of chlorophyll $a$ have in most cases decreased. These effects usually appear both during biomanipulation and in the early postmanipulation phase. Nonetheless, we found a great deal of variability among case studies, and there are cases of lakes that did not improve.

\section{Quantitative synthesis}

Summary effect sizes based on datasets of different quality

Quantitative analysis of available data substantiates the observations that concluded our narrative synthesis. According to the meta-analyses summarised in Figure 8, biomanipulation leads to a significant $(\mathrm{p}<0.05)$ increase of water transparency (measured as Secchi depth) and a significant decrease of phytoplankton abundance (measured as concentration of chlorophyll $a$ ) in summer, not only during years when such manipulation is carried out, but also during the first three post-manipulation years.

A large proportion - $85 \%$ or more - of all available Secchi depth and chlorophyll $a$ effect sizes (i.e. the data presented in Figure 7) are meta-analysable in the sense that we have access to information on variation and sample sizes. Our meta-analyses of these data indicate that, on the average, Secchi depths are $0.22 \mathrm{~m}$ greater and chlorophyll $a$ concentrations $22 \mu \mathrm{g} / \mathrm{l}$ lower during biomanipulation than before manipulation. The first three years

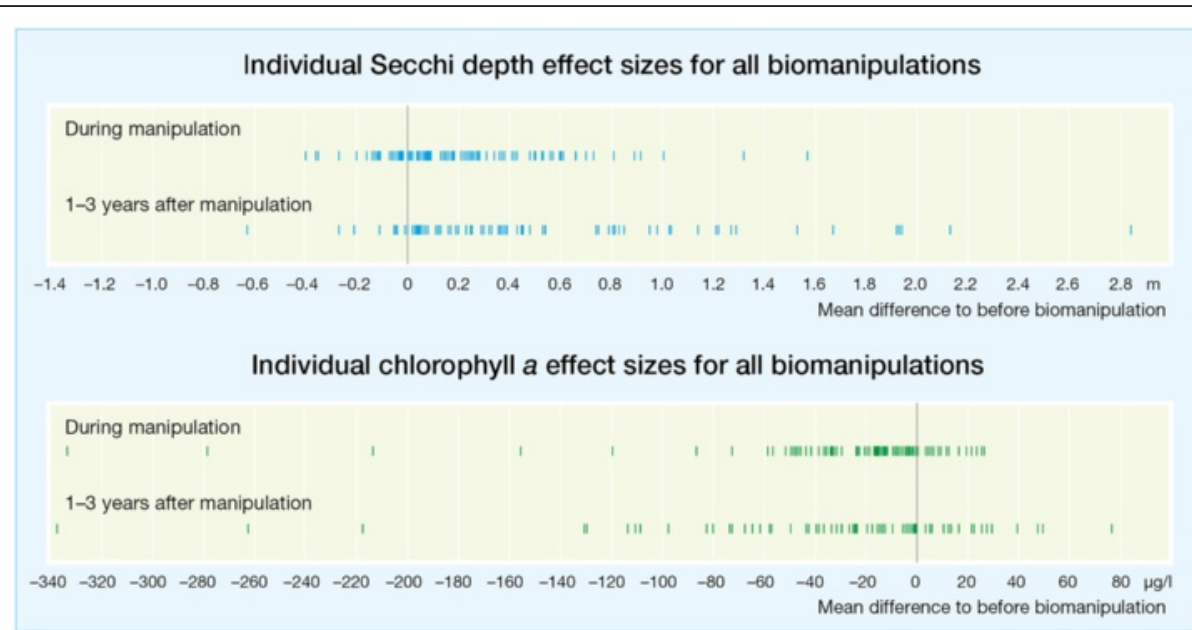

Figure 7 Effect sizes based on mean Secchi depth and chlorophyll $a$ concentration. All biomanipulations with available data are represented in the diagram. 'Before' and 'During' periods have been defined as in Figures 5 and 6 . All Secchi depth and chlorophyll $a$ data in this and the following figures and tables are based on summer means. 
Table 4 Summary effect sizes based on the selected dataset (mean differences to before manipulation)

\begin{tabular}{lll}
\hline & Mean & 95\% C.I. \\
\hline Secchi depth during manipulation $(\mathrm{m})$ & 0.22 & $0.11-0.33$ \\
Secchi depth 1-3 years after manipulation $(\mathrm{m})$ & 0.47 & $0.23-0.70$ \\
Chlorophyll a during manipulation $(\mu \mathrm{g} / \mathrm{l})$ & -30 & $-42--17$ \\
Chlorophyll a 1-3 years after manipulation $(\mu \mathrm{g} / \mathrm{l})$ & -33 & $-52--14$ \\
\hline
\end{tabular}

after biomanipulation, Secchi depths are $0.46 \mathrm{~m}$ greater and chlorophyll $a$ concentrations $30 \mu \mathrm{g} / \mathrm{l}$ lower than before manipulation, again based on averages of all metaanalysable data. All these summary effect sizes are statistically significant (see the topmost row in Figure 8 and pp. 1-4 in Additional file 10).

Calculation of interannual variation has enabled us to include some water-quality data in meta-analyses even in cases when there was no useful information on within-year variation (see Methods). However, there are indications that the within-year variation of water quality differs from the interannual variation. In 13 lakes where we have multiple data per summer season for at least 5 years within a pre-, during- or post-manipulation period, the within-year Secchi depth variation during these periods was on average 56\% larger than the interannual variation. For chlorophyll $a$ data, the corresponding difference was 68\%. There are also some differences between summary effect sizes based on single vs. multiple data per year (i.e. data with interannual variation only and data with within-year variation over one or several years, respectively), as shown on rows 2 and 3 in Figure 8 (and pp. 5-8 in Additional file 10). The difference is statistically significant for Before/During comparisons of chlorophyll $a$, but while the summary effect size is smaller for single- than for multiple-per-year chlorophyll $a$ data, the reverse applies to Secchi depth data.

Moreover, we have classified outcomes of about a quarter of the included biomanipulations as confounded since additional interventions or disturbances took place during, just before or just after the main biomanipulation (see Additional file 7: Table B). Confounded effect sizes tend to be smaller than nonconfounded ones (see Figure 8, rows 4 and 5, and Additional file 10, pp. 9-12).

In order to reduce the risk of bias, we have based most of the further quantitative analysis on the 'selected dataset' from which single data per year and confounded data have been excluded (see the Methods section). Summary effect sizes calculated using the selected dataset are shown in Table 4, in Figure 8 (bottom row) and in Additional file 10 (pp. 17-18). For Secchi depth, they are almost identical to summary effect sizes based on all meta-analysable data, whereas for chlorophyll $a$ they are somewhat larger, but not significantly so.

Alternatively, we could have defined a high-quality dataset by including effect sizes only for those biomanipulations where data were categorised as having low susceptibility to bias (see Methods). Summary effect sizes based on such data are very similar to those based on the selected dataset, as indicated by the two bottommost rows in Figure 8 (and pp. 13-16 in Additional file 10).

The Secchi depth and chlorophyll $a$ effect sizes reported above are all based on mean differences. We also explored the consequences of exchanging mean differences for mean log ratios when analysing the selected dataset, but this did not alter the main results - Secchi depth increases and chlorophyll $a$ decreases all remained significant.

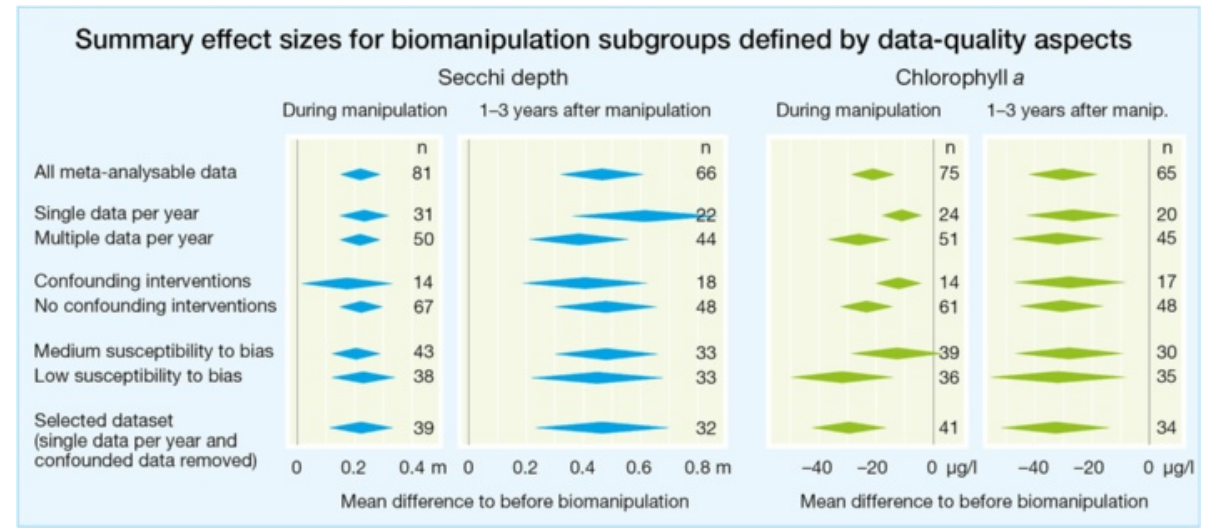

Figure 8 Summary effect sizes for biomanipulation subgroups defined by different aspects of data quality. The diamond-shaped symbols show means based on meta-analysable Secchi depth and chlorophyll $a$ data (with 95\% confidence intervals indicated by the widths of the symbols). The number of individual effect sizes ( $\mathrm{n}$ ) is indicated for each subgroup. 'Before' and 'During' periods have been defined as in Figures 5 and 6 . Forest plots showing all individual effect sizes are presented in Additional file 10. 


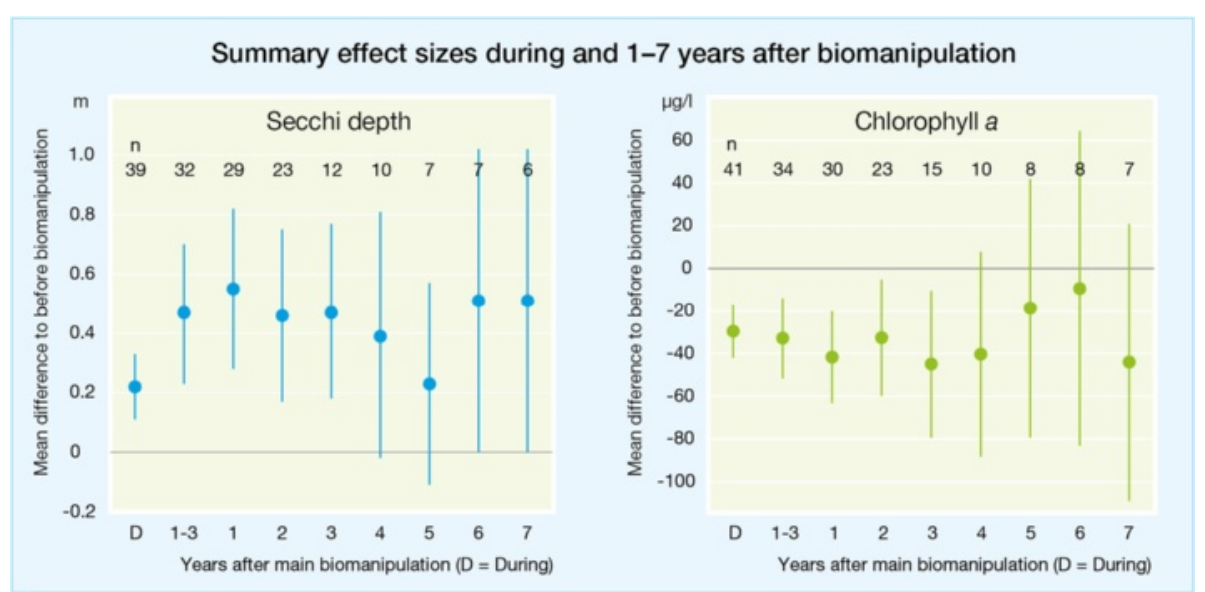

Figure 9 Summary effect sizes during and 1-7 years after biomanipulation. Means based on the selected dataset are shown during manipulation (D) and 1-3 years after manipulation, and also for each of the first 7 years after manipulation. Vertical lines indicate $95 \%$ confidence intervals. Numbers of biomanipulations with data are indicated in the upper part of the panels. 'Before' and 'During' periods have been defined as in Figures 5 and 6. Forest plots showing all individual effect sizes are presented in Additional file 10 (pp. 19-22).

\section{Persistence of biomanipulation effects}

Summary effect sizes for individual post-manipulation years show that four years or more after biomanipulation, the effects on Secchi depth and chlorophyll $a$ are no longer significant, or just barely significant (see Figure 9).
This may at least partly be due to the decrease of available information over time (see the number of observations in the upper part of Figure 9, and also the distribution over time of all individual meta-analysable effect sizes in Figure 10).

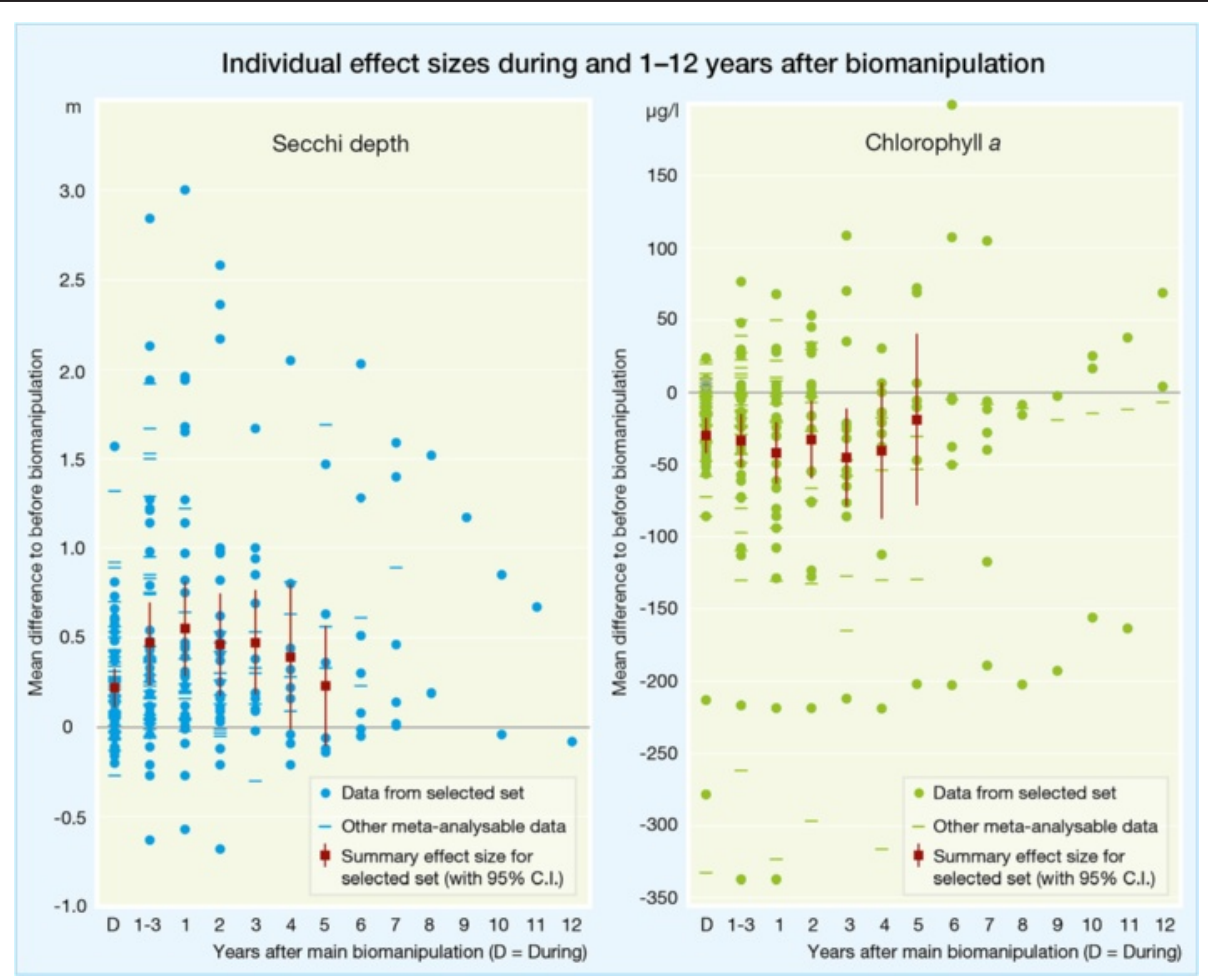

Figure 10 Individual effect sizes during and 1-12 years after biomanipulation. Here, distributions of effect sizes based on the selected dataset can be compared with those of other meta-analysable data. Individual effect sizes are shown during manipulation (D) and 1-3 years after manipulation, and also for each of the first 12 years after manipulation. Summary effect sizes based on the selected dataset are shown during manipulation and for the first 5 years after manipulation. 'Before' and 'During' periods have been defined as in Figures 5 and 6. 
Another factor that most likely contributes to the variation of summary effect sizes in Figure 9 is that the data are based on different sets of manipulations in different years. In Figure 11, therefore, we present individual effect sizes for biomanipulation cases where long and more or less unbroken time series are available. These data, too, indicate that manipulation effects may last for a considerable number of years, in some cases ten years or more.

It is difficult to draw any general conclusions from these results, however, since a selection effect is involved. In this review, we followed the water quality of manipulated lakes only as long as no new mass removals of fish or other large-scale interventions were carried out. In many cases, though, lake managers repeated the biomanipulation after a few years since the water quality had then deteriorated. After the renewed intervention, such lakes no longer appear in our data. This means that lakes where manipulation effects have been more persistent than average are likely to be overrepresented in the set of biomanipulations for which we have data over many years.

Moreover, in 6 of the 13 cases represented in Figure 11 (panels at right), the main biomanipulation was followed up with other interventions (e.g. stocking or aeration) over several years, and this may have contributed to the persistence of the water-quality effects.

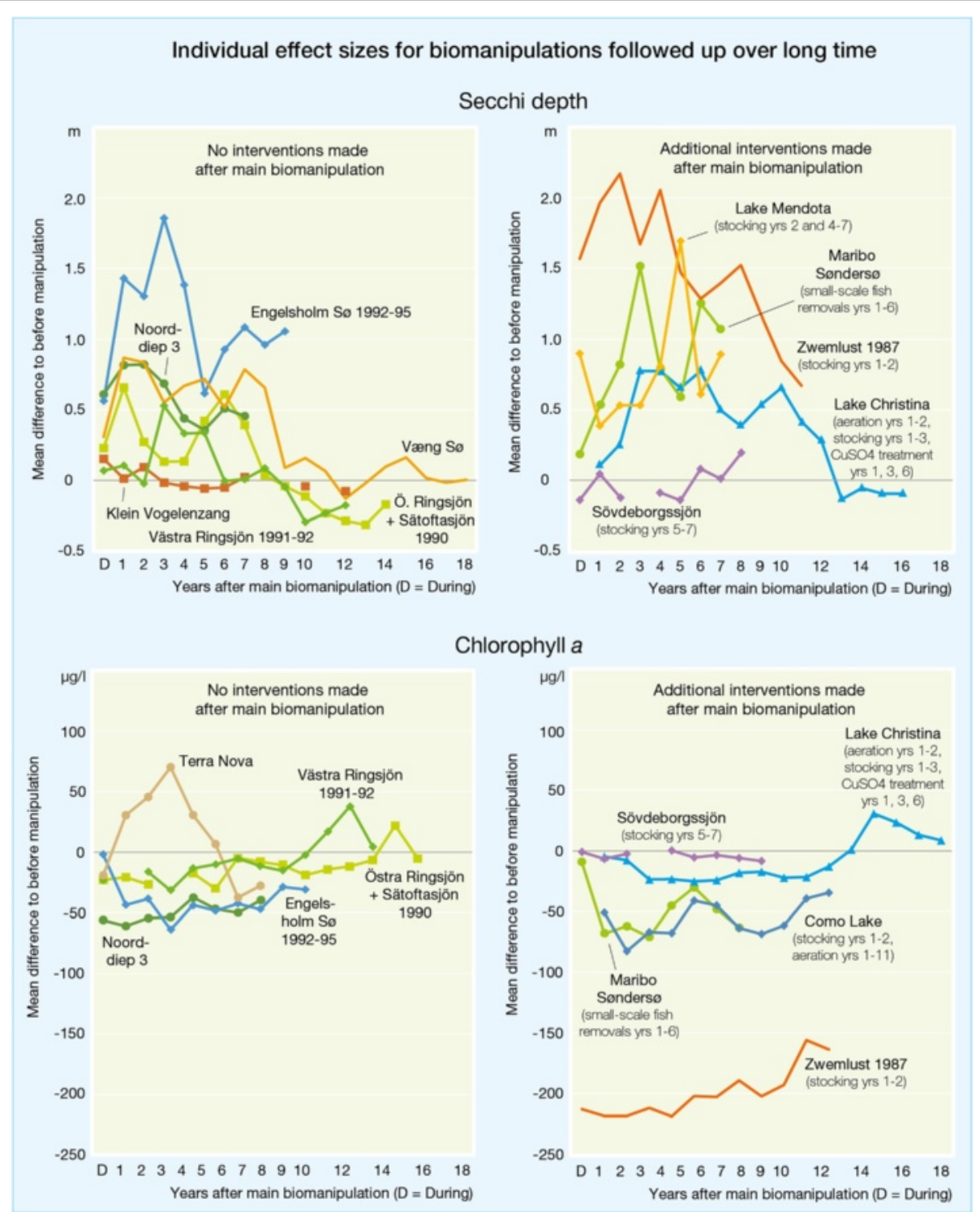

Figure 11 Individual effect sizes for biomanipulation cases where long time series are available. The effect sizes shown for Engelsholm Sø, Lake Christina, Maribo Søndersø, Væng Sø, Västra Ringsjön and Östra Ringsjön + Sätoftasjön are based on single data per year. The data in the panels at right may also have been influenced by interventions performed after the main biomanipulation, as detailed in the figure. 


\section{Effects on cyanobacteria abundance}

Biomanipulation can also reduce the abundance of cyanobacteria (see Figure 12). Based on data for six biomanipulations in the selected set, the cyanobacteria abundance in summer decreased by an average of $84 \%$ from the premanipulation period to the first three years after manipulation. The available post-manipulation data is very limited, however, and the summary effect size remained significant only during the first year after manipulation.

\section{Effects of planktivore/benthivore removal vs. piscivore stocking}

The biomanipulations that we have studied include removals of planktivorous and/or benthivorous fish as well as stockings of piscivorous fish, and also cases where these two approaches have been combined.

We have found clear contrasts between the waterquality effects of different kinds of biomanipulation (see Figure 13). Removal of planktivores/benthivores led to increased Secchi depth and decreased chlorophyll $a$ concentration, both during intervention and in the first three post-intervention years, and regardless of whether the removal was combined with piscivore stocking or not. With one exception (Secchi depth 1-3 years after removal plus stocking), the effects were all significant. By contrast, manipulation based on piscivore stocking alone had no significant effect on Secchi depth or chlorophyll a concentration, neither during nor after the intervention.

\section{Biomanipulation effects in relation to lake properties and intervention strength}

The studies we have reviewed and analysed indicate that removal of planktivorous and/or benthivorous fish is capable of increasing water transparency and decreasing the amount of phytoplankton in lakes. However, the size of these effects varies both with lake properties and with intervention strength.

In Table 5, lakes that responded to biomanipulation (i.e. where water quality improved significantly) are compared with 'unresponsive' lakes (i.e. lakes where water quality did not change significantly, or even deteriorated). Lakes where water transparency was significantly larger after manipulation than before tended to be smaller and have shorter retention times than lakes where transparency did not improve. Similar tendencies can be seen in lakes where the chlorophyll $a$ concentration was significantly lower after manipulation than before. These lakes also had higher pre-manipulation concentrations of total phosphorus (TP) than lakes where the chlorophyll level did not decrease.

We based this analysis on the selected dataset, but manipulations solely consisting of piscivore stocking were

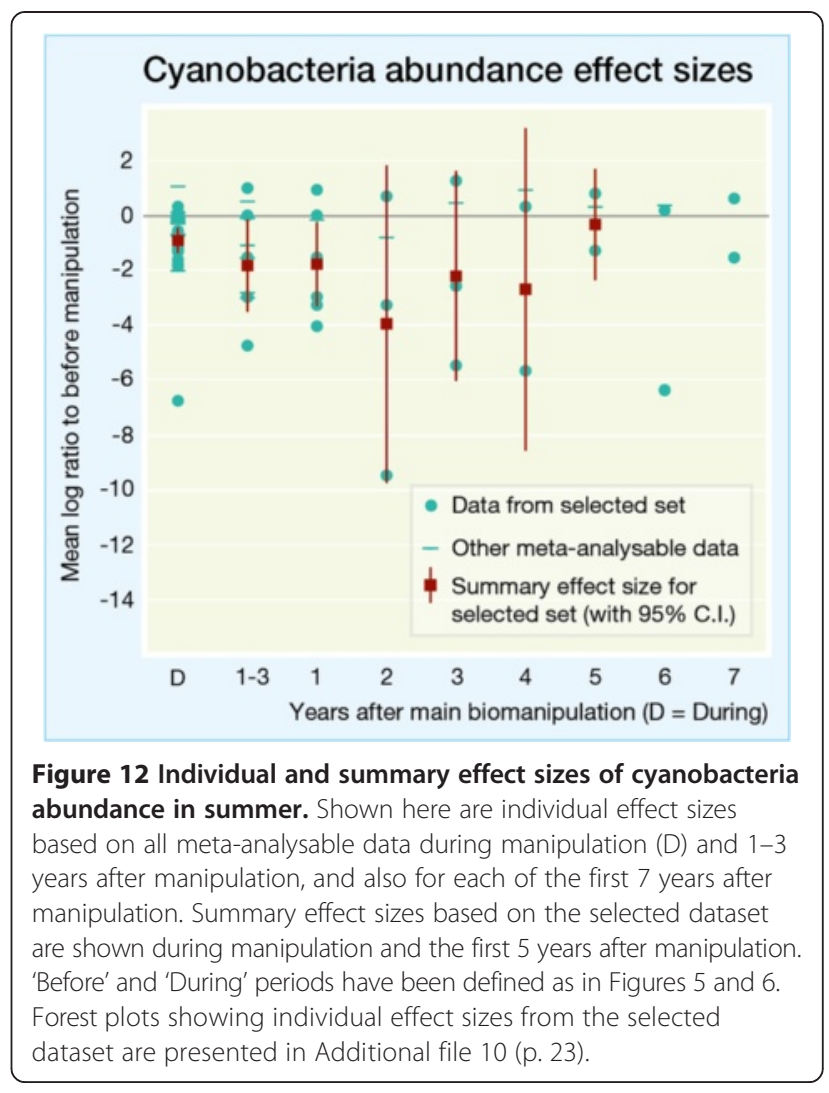

excluded, since we had found no evidence that such treatment improves water quality. This means that the analysis was based on a relatively limited amount of data, and none of the differences between responsive and unresponsive lakes was significant.

Meta-regression model selection showed that the effect of biomanipulation on chlorophyll $a$ levels depends on the amount of fish removed, on the combination of the area and pre-manipulation TP concentration of the lake as represented by PC1 (see Methods), as well as on the interaction between these two variables (Table 6 and Additional file 11).

The selected models for chlorophyll $a$ concentration during and after biomanipulation were both strongly supported (with AICc values more than 15 units less than the next best models; cf. [44]). The mean decrease of the chlorophyll a concentration was greater during biomanipulation in lakes where fish removal was more intense, and it was greater both during and after biomanipulation in lakes that were small and/or had high pre-manipulation TP concentrations (i.e. small values of PC1; Table 6). These relations also tended to reinforce each other - higher intensity of fish removal had a stronger effect on the chlorophyll $a$ concentration in lakes that were small and/or had high pre-manipulation TP concentrations (as shown by the sign of the interaction term between between PC1 and fish removal; Table 6), both during and after biomanipulation. 
Table 5 Comparison of responsive and unresponsive lakes

\begin{tabular}{|c|c|c|c|c|c|c|}
\hline & \multicolumn{3}{|c|}{ Responsive lakes (significant improvement) } & \multicolumn{3}{|c|}{ Unresponsive lakes (no significant improvement) } \\
\hline & Mean & 95\% C.I. & $\mathrm{n}$ & Mean & 95\% C.I. & $\mathbf{n}$ \\
\hline \multicolumn{7}{|c|}{ Response: Secchi depth 1-3 years after manipulation (vs. before manipulation) } \\
\hline Lake area (ha) & 18 & $10-32$ & 19 & 40 & $14-119$ & 8 \\
\hline Mean depth (m) & 1.7 & $1.3-2.2$ & 19 & 1.8 & $1.3-2.5$ & 8 \\
\hline Retention time (days) & 171 & $63-461$ & 11 & 409 & $221-754$ & 5 \\
\hline Pre-manipulation TP $(\mu \mathrm{g} / \mathrm{l})$ & 144 & $93-223$ & 19 & 127 & $62-260$ & 6 \\
\hline Mean atmospheric temperature $\left({ }^{\circ} \mathrm{C}\right)$ & 8.0 & $7.0-9.0$ & 19 & 7.8 & $6.4-9.2$ & 8 \\
\hline Duration of main manipulation (yr) & 2.1 & $1.6-2.5$ & 19 & 2.4 & $1.5-3.3$ & 8 \\
\hline Fish removal (kg/ha) & 233 & $160-338$ & 18 & 251 & $198-317$ & 7 \\
\hline Fish removal (kg/ha/yr) & 124 & $84-183$ & 18 & 119 & $70-203$ & 7 \\
\hline Fish stock depletion (\%) & 56 & $37-76$ & 13 & 40 & $17-62$ & 5 \\
\hline \multicolumn{7}{|c|}{ Response: Chlorophyll a 1-3 years after manipulation (vs. before manipulation) } \\
\hline Lake area (ha) & 12 & $5-36$ & 12 & 35 & $14-86$ & 15 \\
\hline Mean depth (m) & 1.3 & $1.1-1.6$ & 11 & 1.8 & $1.4-2.4$ & 15 \\
\hline Retention time (days) & 78 & $22-275$ & 4 & 210 & $103-428$ & 9 \\
\hline Pre-manipulation TP $(\mu \mathrm{g} / \mathrm{l})$ & 196 & $120-322$ & 12 & 126 & $83-190$ & 13 \\
\hline Mean atmospheric temperature $\left({ }^{\circ} \mathrm{C}\right)$ & 8.1 & $7.1-9.1$ & 12 & 8.2 & $7.5-8.9$ & 15 \\
\hline Duration of main manipulation (yr) & 2.0 & $1.4-2.6$ & 12 & 2.0 & $1.5-2.5$ & 15 \\
\hline Fish removal (kg/ha) & 250 & $149-420$ & 11 & 272 & $184-403$ & 12 \\
\hline Fish removal (kg/ha/yr) & 137 & $74-252$ & 11 & 140 & $98-202$ & 12 \\
\hline Fish stock depletion (\%) & 78 & $56-99$ & 7 & 58 & $42-75$ & 12 \\
\hline
\end{tabular}

Data are based on the selected dataset, with stocking-only interventions excluded. Lake areas, mean depths, retention times, pre-manipulation TP concentrations and fish removals were log-transformed before calculation of means and confidence intervals, and then back-transformed.

Lake characteristics, in terms of area and pre-manipulation TP, clearly influence the effect of biomanipulation on chlorophyll $a$ concentration both during and after manipulation, as the model with fish removal alone had a $\Delta$ AICc $>20$ (Table 6).

High intensity of fish removal also corresponded to greater increases in water transparency (measured as Secchi depth) during biomanipulation (Table 6). In contrast to chlorophyll $a$ concentrations, Secchi depth changes during biomanipulation were not related to lake properties (as the best model included only the intensity of fish removal). Several models of water transparency after biomanipulation received similar level of support (Table 6). The most supported model included only lake properties (PC1), but the support for the null (intercept only) model was almost as high $(\triangle \mathrm{AICC}=1.15)$. The model with only fish removal also had a $\triangle$ AICc $<2$, showing that Secchi depth after biomanipulation may be explained either by lake properties or intervention strength.

For the purposes of exploration and illustration, we also performed meta-regressions with single effect modifiers (see Figure 14 and Additional file 12). These showed the improvement of water-quality caused by biomanipulation to decrease with lake area and to increase with pre-manipulation TP concentration (although not significantly so for Secchi depth after manipulation). Moreover, the effect of biomanipulation on chlorophyll $a$ decreased significantly with increasing retention time. We also found that biomanipulation effects on water quality increased with fish removals as expressed per hectare and year (significantly so for Secchi depth and chlorophyll $a$ during but not after manipulation; see Figures 14 and 15) and with the depletion of fish stocks (but significantly so only for Secchi depth after manipulation; see Figures 14 and 16).

Using effect sizes based on mean log ratios instead of mean differences produces similar results, although relations between Secchi depth changes and effect modifiers tend to become more significant, whereas the reverse applies to chlorophyll $a$ changes.

\section{Tests for possible publication bias}

Earlier reviews have found certain evidence of publication bias in the literature about biomanipulation effects - seemingly, negative results have not been reported to the same extent as positive experiences [8]. In this review, we tested our selection of studies for publication bias using funnel plots (see Additional file 13). These plots do indicate that studies that have high precision (i.e. low standard error, usually due to a large number of 
Table 6 AICc model selection

\begin{tabular}{|c|c|c|c|c|c|}
\hline Intercept & PC1 & Fish removal & PC1 x Fish removal & AICc & $\triangle \mathrm{AICC}$ \\
\hline \multicolumn{6}{|c|}{ Chlorophyll a during manipulation } \\
\hline+1 & -1 & -1 & +1 & 245.06 & 0.00 \\
\hline+1 & +1 & -1 & & 260.91 & 15.85 \\
\hline+1 & & -1 & & 267.20 & 22.13 \\
\hline-1 & +1 & & & 271.04 & 25.98 \\
\hline-1 & & & & 283.14 & 38.07 \\
\hline \multicolumn{6}{|c|}{ Chlorophyll a 1-3 years after manipulation } \\
\hline-1 & -1 & +1 & +1 & 201.11 & 0.00 \\
\hline+1 & +1 & -1 & & 216.44 & 15.33 \\
\hline-1 & +1 & & & 223.51 & 22.41 \\
\hline+1 & & -1 & & 223.87 & 22.76 \\
\hline-1 & & & & 234.28 & 33.17 \\
\hline \multicolumn{6}{|c|}{ Secchi depth during manipulation } \\
\hline-1 & & +1 & & 24.70 & 0.00 \\
\hline-1 & -1 & +1 & & 27.67 & 2.97 \\
\hline-1 & +1 & +1 & -1 & 27.81 & 3.11 \\
\hline+1 & -1 & & & 28.94 & 4.24 \\
\hline+1 & & & & 31.26 & 6.56 \\
\hline \multicolumn{6}{|c|}{ Secchi depth 1-3 years after manipulation } \\
\hline+1 & -1 & & & 44.71 & 0.00 \\
\hline+1 & & & & 45.87 & 1.15 \\
\hline-1 & & +1 & & 46.11 & 1.39 \\
\hline+1 & +1 & -1 & -1 & 46.98 & 2.26 \\
\hline+1 & -1 & +1 & & 47.18 & 2.47 \\
\hline
\end{tabular}

The explanatory variables included in each model are indicated by +1 or -1 , which shows the sign of their effects. AICc values are given, and also the difference in AICc between each model and the model with the lowest AICc. Models are arranged according to AICC value. Data are based on the selected set of effect sizes, with stocking-only interventions excluded. The output of the most supported models is presented in Additional file 11.

observations) generally report effect sizes closer to zero than studies with lower precision. This asymmetry, which suggests the possibility of publication bias, is clearly visible when all studies in the review are considered, especially among Secchi depth data, but it also appears in the selected dataset.

\section{Discussion}

Our review and meta-analysis show that biomanipulation of lakes increases Secchi depth and decreases chlorophyll $a$ concentration (Figure 8, Table 4). Nonetheless, there is considerable variability among lakes, which is discussed further below. Within this variability some significant patterns are evident: (1) The effects of biomanipulation are significant during and 1-3 years after treatment (Figures 9, 10 and 11). (2) Removal of planktivores and benthivores, with or without stocking of piscivores, is capable of improving water clarity, but piscivore stocking alone has no significant effect of that kind (Figure 13). (3) Lakes that do not respond to biomanipulation tend to have longer water residence times and lower percentage depletion of stocks of planktivorous and benthivorous fish (Table 5). There is also a tendency for non-responding lakes to be larger in surface area, although there is a wide range in lake area for responding and non-responding lakes. (4) Effects of biomanipulation on chlorophyll $a$ are significantly stronger in cases where fish removal is more intense and where pre-manipulation TP is higher and/or the lake area smaller.

Cyanobacteria are of special interest in lake management because of their potential toxicity and their capacity to form noxious scums on the lake surface. The case studies that reported responses of cyanobacteria were fewer than those that reported Secchi depth or chlorophyll $a$. Nonetheless, we found that biomanipulation significantly decreased cyanobacteria concentrations for up to three years after treatment (Figure 12).

Our review improves in several ways on previous reviews of biomanipulation. We obtained all of the literature that was available on a range of literature databases and systematically screened for useful studies. As a result our analyses included a large number of case studies from many parts of the world. We used a consistent, repeatable process to screen published reports for inclusion in further analyses. We then analysed the data using standard methods of meta-analysis [39].

Several previous reviews have concluded that biomanipulation is successful under some conditions $[7,11,16,29,30,45,46]$. These reviews reach various conclusions about the factors that lead to success or failure of biomanipulations. Some of the variability among reviews may be explained by differences in datasets available at the times the papers were written, or differences in the process for selecting papers for review.

As noted in Table 7, our findings are consistent with the conclusion of a number of previous reviews that substantial fish removals are needed for successful biomanipulation [11,16,29]. According to our metaanalysis, removal of benthivores and planktivores (with or without stocking of piscivores) has significant effects on water quality effects. By contrast, and in line with conclusions by Søndergaard et al. [7], our findings also suggest that piscivore stocking alone does not affect water quality as measured by Secchi depth and chlorophyll $a$.

On the other hand, our findings on the importance of lake properties differ from conclusions of some previous reviews (Table 7). Several authors point out that biomanipulation is mainly successful in shallow and/or small lakes $[30,46]$, while our results show that successful and failed biomanipulations have occurred in overlapping ranges of 


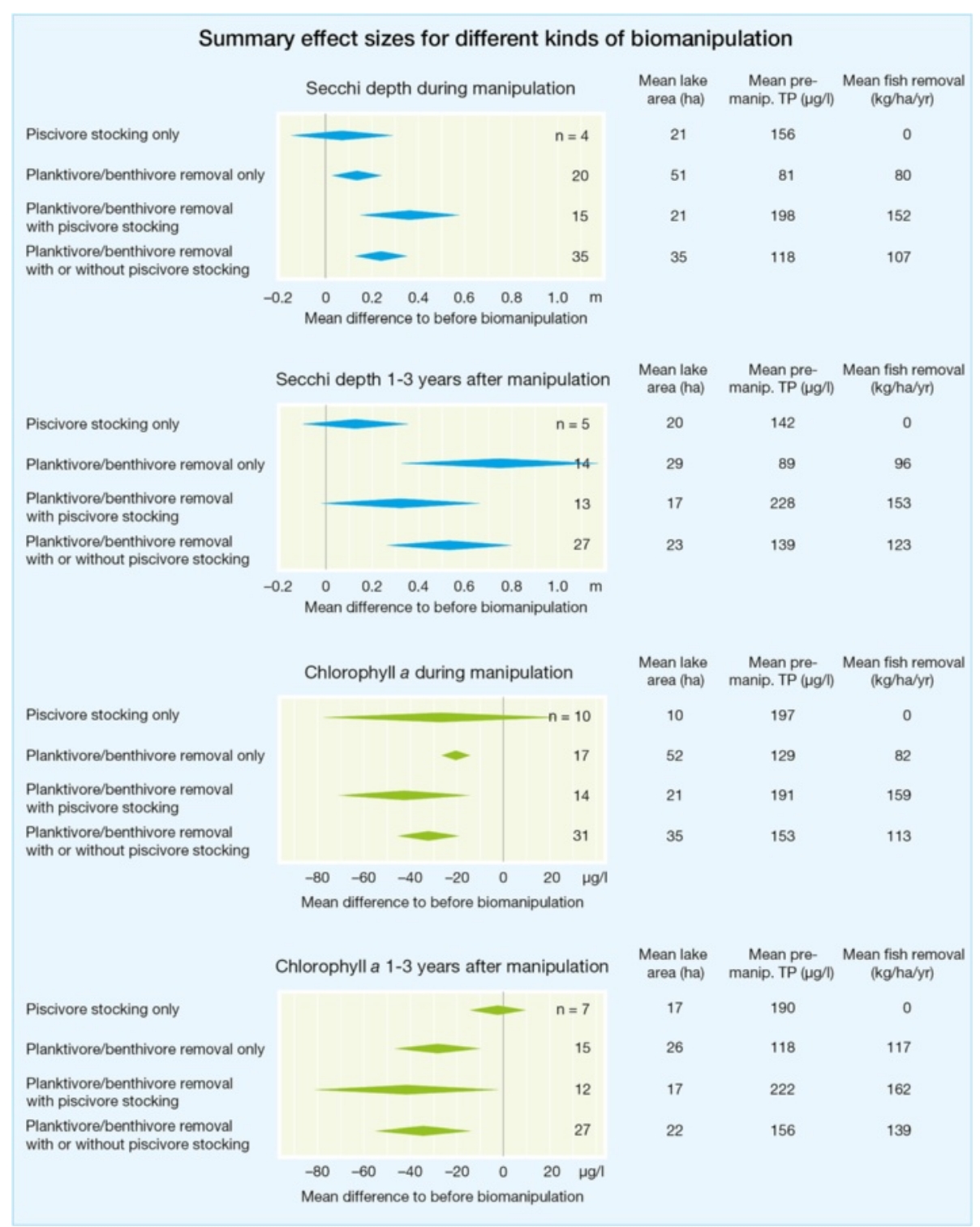

Figure 13 Summary effect sizes for biomanipulations based on piscivore stocking and/or planktivore/benthivore removal. The

diamond-shaped symbols show means of Secchi depth and chlorophyll a data from the selected set (with 95\% confidence intervals indicated by the widths of the symbols). In the bottom row of each panel, data presented in the second and third rows have been pooled. Forest plots showing all individual effect sizes are presented in Additional file 10 (pp. 24-27). Subgroup means of potential effect modifiers (lake area, pre-manipulation total phosphorus concentration (TP) and fish quantities removed per hectare and year) are shown at right. These data were log-transformed before the calculation of means, and then back-transformed.

lake mean depths and surface areas (Table 5, Figure 14). Nonetheless, we found that biomanipulation effect size declines with increasing lake area. We found that lakes with higher pre-manipulation TP respond more strongly to biomanipulation. Thus our findings do not support the conclusion that biomanipulation will not work if TP is too high [46].

Some of the studies included in this review reported on effects of biomanipulation that persisted even when the system would have been expected to return to initial conditions in the absence of alternative stable states $[17,19]$. The number of lakes with long-lasting biomanipulation effects was small, however (see next section), and data that would allow an analysis of mechanisms related to alternative stable states (such as the development of macrophytes over time) were lacking in most of these cases. We therefore refrain from attempting any such analysis in this report. 


\section{Relations between biomanipulation effects,} lake properties and intervention strength

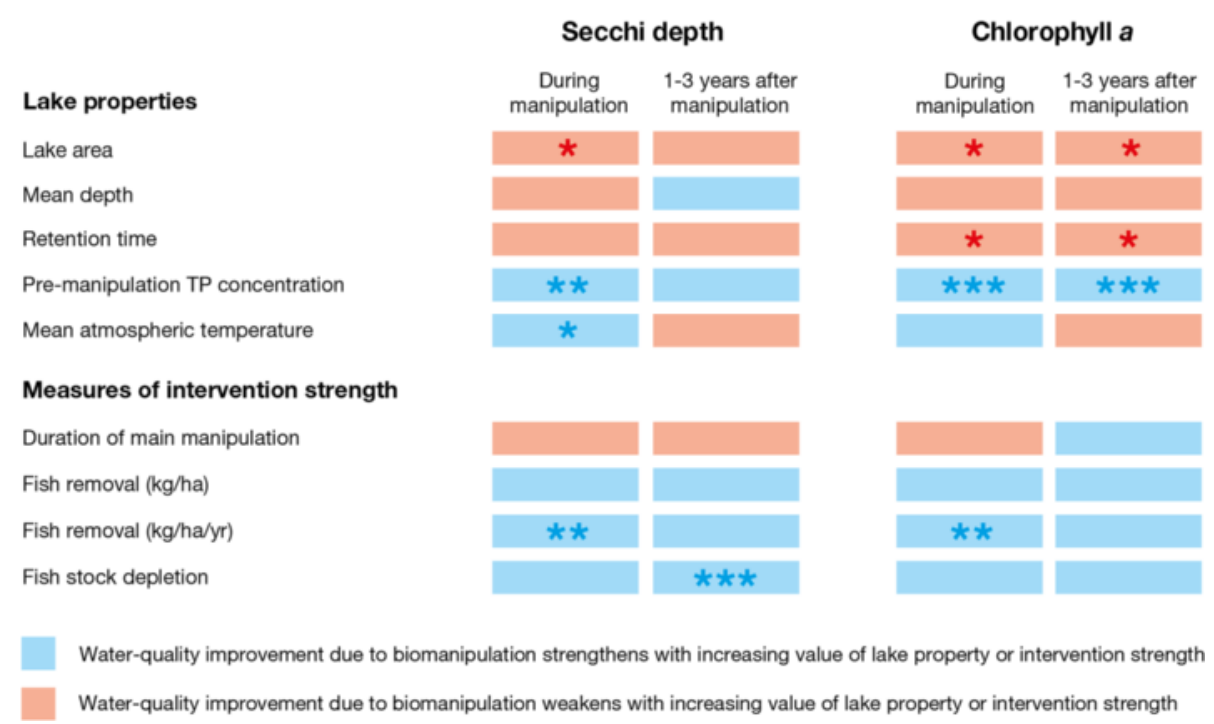

Figure 14 Relations between biomanipulation effects, lake properties and measures of intervention strength. Colours indicate whether changes of lake properties or intervention strength tend to strengthen or weaken the ability of biomanipulation to improve water quality (i.e. its ability to increase the Secchi depth or decrease the chlorophyll a concentration). Some of the meta-regressions summarised here are also presented in Additional file 12. The significance of the relations is indicated with one $(p<0.05)$, two $(p<0.01)$ or three $(p<0.001)$ asterisks. Lake areas, mean depths, retention times, pre-manipulation TP concentrations and fish removals were log-transformed. All analyses used the selected dataset with stocking-only biomanipulations excluded.

\section{Reasons for heterogeneity}

The variability among lakes in responses to biomanipulation has many dimensions, some of which can be illuminated using our dataset. It must be noted that no review of biomanipulation, including ours, has access to datasets in which important co-variates such as lake area, phosphorus loading, and magnitude of fish removal are statistically independent and sampled continuously from pre-manipulation until effect of the manipulation are no longer discernible. In the absence of such datasets, any evaluation of co-variate effects is provisional. Nonetheless, several statistically significant effects of co-variates should be discussed here.

Data quality appears to influence outcomes, especially the response of chlorophyll $a$ (Figure 8). Therefore we focused on a high-quality 'selected' dataset that excludes studies based on a single datum per year and studies that confound biomanipulation with other types of manipulations.

Effects of biomanipulation are detectable statistically up to 3 years after the manipulation in the meta-analysis (Figures 9 and 10). Biomanipulation studies performed over longer periods are rare, however, and the variation of summary effect sizes increases as the number of lakes included in the meta-analyses goes down with the number of years elapsed after intervention (Figure 9). No statistically significant effect can therefore be found 4 years or more after biomanipulation, but the mean effect sizes show no obvious signs of diminishing even up to 7 years after intervention (Figure 9). In certain lakes the effects of biomanipulation last considerably longer, up to 10 or more years (Figure 11). Long-lasting effects were observed in deep stratified lakes (e.g. Mendota) as well as shallow well-mixed ones (e.g. Zwemlust). These results are in line with findings by Gulati and Van Donk [45] and Søndergaard et al. [7,16], which suggest that effects of biomanipulation can last up to 6-10 years but that water clarity eventually degrades in most cases.

Physical and chemical characteristics of lakes that affect biomanipulation success include lake area, water retention time, and pre-manipulation TP (Table 5, Figure 14). It is easier to remove large fractions of the benthivore and planktivore stocks from smaller lakes, which may be one reason why these tend to show stronger responses to biomanipulation. Lakes with longer retention times (i.e. slowly-flushed lakes) are less affected by biomanipulation, maybe because fish removal effects are counteracted by a higher degree of internal phosphorus loading. Lakes with high pre-manipulation TP show stronger responses to biomanipulation, especially when it comes to chlorophyll $a$. Initial chlorophyll concentrations are often very high in highly eutrophied lakes, which could mean that a large chlorophyll reduction (in absolute terms) is easier to achieve there than in less 


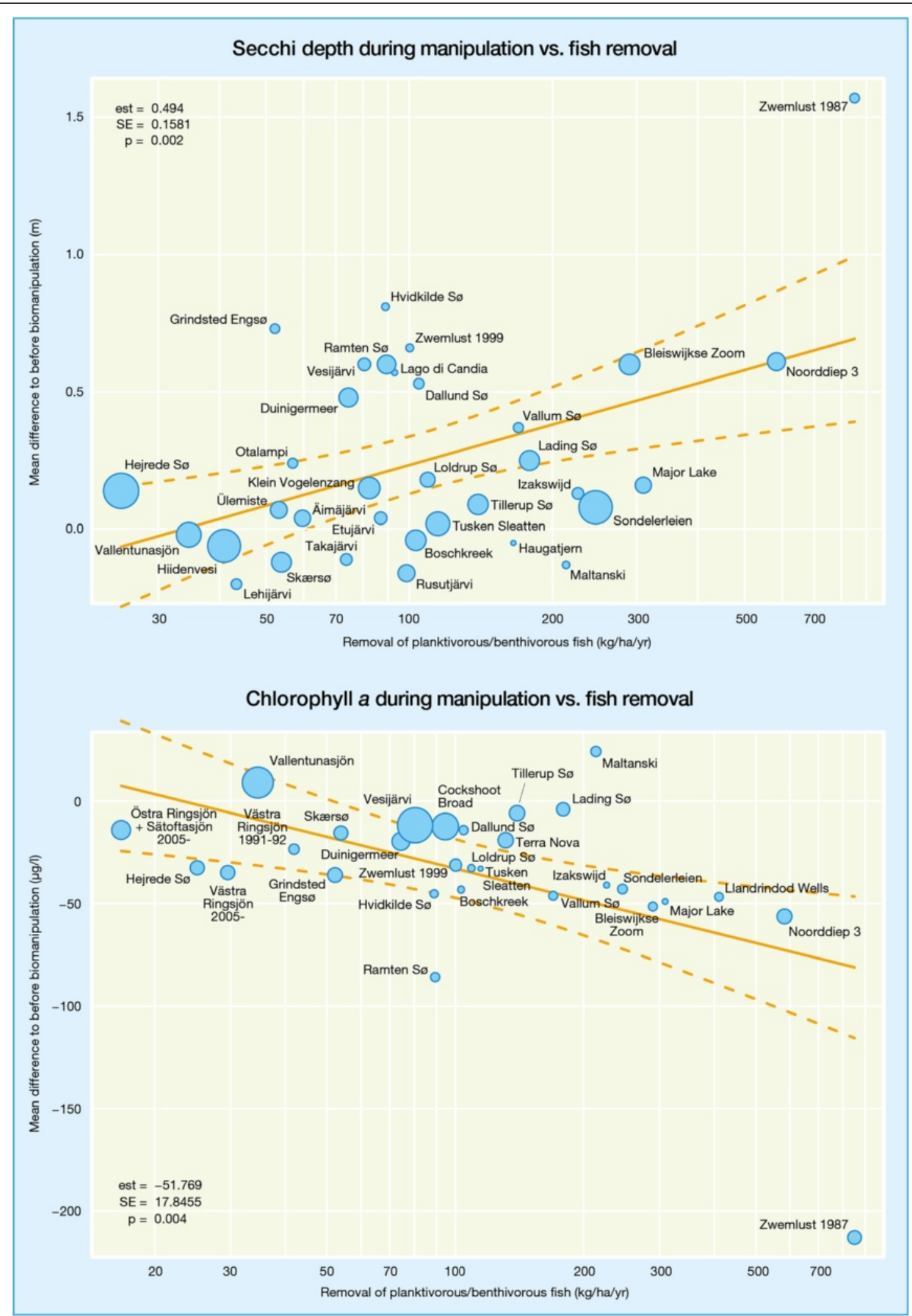

Figure 15 Meta-regressions of Secchi depth and chlorophyll $a$ during manipulation vs. fish removal. Each symbol represents one biomanipulation. Symbol sizes indicate statistical weights based on inverse variances. 
Table 7 Overview of conclusions in this and earlier reviews

\begin{tabular}{|c|c|c|}
\hline $\begin{array}{l}\text { Conclusions in } \\
\text { earlier reviews }\end{array}$ & Ref. & $\begin{array}{l}\text { Supported } \\
\text { by this } \\
\text { review }\end{array}$ \\
\hline $\begin{array}{l}\text { Planktivore/benthivore removal increases water } \\
\text { transparency }\end{array}$ & {$[7,11,16,29]$} & Yes \\
\hline $\begin{array}{l}\text { Planktivore/benthivore removal decreases } \\
\text { chlorophyll a }\end{array}$ & {$[7,11,16,29]$} & Yes \\
\hline $\begin{array}{l}\text { Planktivore/benthivore removal decreases } \\
\text { cyanobacteria abundance }\end{array}$ & {$[16,29]$} & Yes \\
\hline $\begin{array}{l}\text { Increased planktivore/benthivore removal } \\
\text { increases biomanipulation effects }\end{array}$ & {$[11,16,29]$} & Yes \\
\hline $\begin{array}{l}\text { Piscivore stocking is less efficient than } \\
\text { planktivore/benthivore removal }\end{array}$ & {$[7,30]$} & Yes \\
\hline Biomanipulation is more efficient in shallow lakes & {$[30,46]$} & No \\
\hline Biomanipulation is more efficient in small lakes & {$[30]$} & Yes \\
\hline $\begin{array}{l}\text { Biomanipulation is less efficient in lakes with } \\
\text { high pre-manipulation TP }\end{array}$ & [46] & No \\
\hline
\end{tabular}

eutrophic lakes with lower pre-manipulation chlorophyll levels. These speculations are interesting topics for future research but cannot be resolved here.

Intervention strength has variable but detectable effects on the response of lakes to biomanipulation (Table 5, Figures 14, 15 and 16). The high variance of fish population estimates may be a factor in the statistical analyses. Uncertainty in the $\mathrm{x}$-axis will decrease the slope of a regression, for example (e.g. Figures 15 and 16). Nonetheless, there may be real effects of intervention strength as also noted in some earlier review papers $[11,16,29,30]$. Lake Zwemlust is an important case study in this regard. Removals of benthivores and planktivores from Zwemlust in 1987 were exceptionally high, and the response to biomanipulation was also large (Figure 15). As noted above, water quality improvements lasted for an exceptionally long time in Zwemlust.

Chlorophyll $a$ and Secchi depth are widely-used measures of water quality. Secchi depth is largely determined by chlorophyll $a$, which is a proxy for phytoplankton abundance. The two variables are inversely related, but the correlation is never perfect. At a given chlorophyll $a$ concentration, the Secchi depth can be higher or lower depending on the concentration of coloured dissolved organic matter, the concentration of inorganic particles suspended in the water, or the particle size distribution of phytoplankton. Therefore we should not expect to obtain completely consistent results for chlorophyll $a$ and Secchi depth responses to biomanipulation. It is worthwhile to examine both indicators.

\section{Review limitations}

We were unable to analyse every aspect of biomanipulation due to limitations of the available data. For example, we were not able to evaluate relations between biomanipulation and biodiversity. Nonetheless it is clear that certain species, such as rooted submerged plants and large-bodied cladocerans such as Daphnia, play a critical role in many

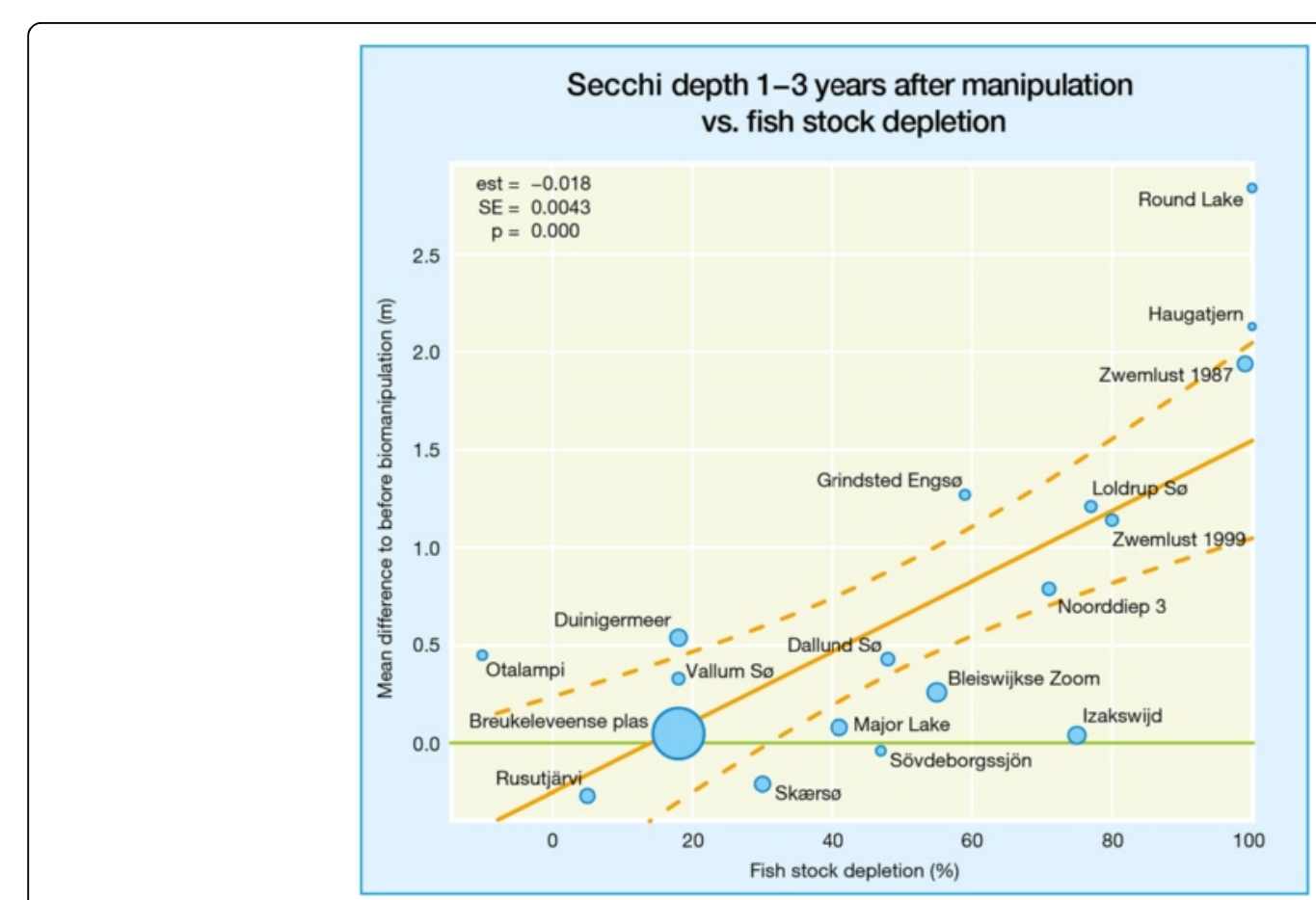

Figure 16 Meta-regression of Secchi depth 1-3 years after manipulation vs. fish stock depletion. Each symbol represents one biomanipulation. Symbol sizes indicate statistical weights based on inverse variances. 
successful biomanipulations. Moreover, the dataset did not allow us to explore the outcomes of piscivore stocking in relation to the species or sizes of stocked fish, although this could have refined our overall conclusion that piscivore stocking alone has no impact on water clarity.

Of the 128 biomanipulations included in this review, more than half (68) were carried out in Denmark or the Netherlands, where most lakes are small, shallow and nutrient-rich. The median area, depth and pre-manipulation TP of the included Danish and Dutch lakes were 23 ha, $1.5 \mathrm{~m}$ and $162 \mu \mathrm{g} / \mathrm{l}$, respectively, whereas the corresponding medians for included lakes in other parts of the world were 78 ha, $2.7 \mathrm{~m}$ and $86 \mu \mathrm{g} / \mathrm{l}$, respectively. This means that the selection of lakes in this review may not be entirely representative of e.g. Swedish, Finnish, Norwegian or North American lakes where biomanipulation has been carried out or could be considered as a future option.

\section{Conclusions}

\section{Implication for policy/management}

Available evidence suggests that biomanipulation is a useful means of improving water quality in eutrophic lakes. Removal of benthivorous and planktivorous fishes (with or without stocking of piscivores) is effective, but piscivore stocking alone is not. More thorough removal of benthivorous and planktivorous fishes increases the effectiveness of biomanipulation in reducing chlorophyll $a$ concentrations. Biomanipulation tends to be particularly successful in relatively small lakes with short retention times and high pre-manipulation phosphorus levels.

Since long-lasting studies are rare, it is difficult to draw conclusions regarding biomanipulation effects more than three years after intervention, but the duration of the effects clearly varies from case to case. In many cases re-treatment is necessary after a few years, but sometimes effects may last a decade or more.

\section{Implication for research}

Our review and meta-analysis uncovered several patterns worthy of further research. More research on the interactive effects of biomanipulation with other lake management tools would be useful and could reveal beneficial combinations of management interventions. The factors that lead to breakdown or persistence of biomanipulation effects in various types of lakes are not yet known. Better understanding could improve ecological theories related to stability and perhaps reveal new information useful for managers.

Our screening process excluded many biomanipulations that could have been analysed had authors provided appropriate data in their original publications. Researchers reporting on the outcomes of such interventions should always publish variances and sample sizes of water quality data, or provide raw data in an electronic appendix. The effects of biomanipulation cannot be assessed properly unless water quality data have been obtained prior to the intervention. Monitoring of water quality should also be continued for at least as long as effects remain evident. Quantitative measures of fish removal, stocking, or biomass changes are necessary and should always be reported.

\section{Additional files}

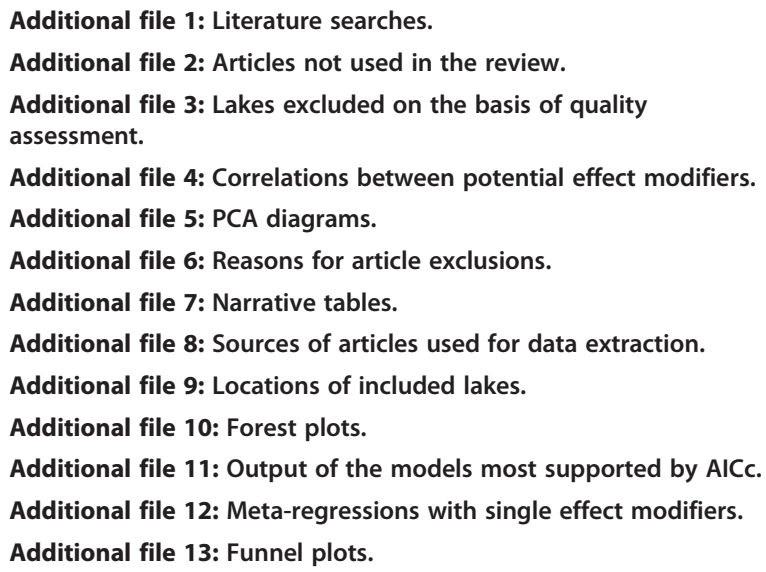

\section{Competing interests}

The authors declare that they have no competing interests.

\section{Authors' contributions}

The team that was appointed by the Mistra Council for Evidence-Based Environmental Management to carry out this review consisted of $\mathrm{CL}, \mathrm{SRC}$, $A G, P L, L P, C S, E V D$. JDMS contributed by performing meta-analyses. All authors participated in the drafting, revision and approval of the manuscript.

\section{Acknowledgements}

The authors are grateful to Corey DeBoom, Richard Nilsson, Terry Noonan, Mikko Olin, Emil Rydin, Björn Tengelin, Gerard ter Heerdt, Egbert van Nes and Sigvard Zachrisson, who all supplied valuable primary data on biomanipulation projects. We would also like to thank Carina Anderberg for finding full-text versions of a large number of publications that we hadn't been able to locate ourselves, and Neal Haddaway for providing advice on how to analyse and report the outcomes of our review.

Moreover, we are grateful for suggestions and comments received from Heléne Annadotter, Stellan Hamrin, Lars-Anders Hansson and Mikko Olin, from three anonymous peer reviewers, and from stakeholders representing the Swedish Ministry of the Environment, the Ministry for Rural Affairs, the Swedish Agency for Marine and Water Management, the Swedish Board of Agriculture, Sportfiskarna (the Swedish association for recreational fishing), the environmental foundation Baltic2020, the municipalities of Täby, Vallentuna and Östhammar, the Rural Economy and Agricultural Society of Kalmar-Kronoberg-Blekinge, the Swedish Society for Nature Conservation, WWF Sweden and the environmental consulting companies Structor Miljöteknik AB and WRS Uppsala AB.

This review was financed by the Mistra Council for Evidence-Based Environmental Management (EviEM). EviEM is funded by the Swedish Foundation for Strategic Environmental Research (Mistra) and hosted by the Royal Swedish Academy of Sciences. The review process has been approved by the EviEM Executive Committee, but the authors are solely responsible for the contents and conclusions of the review.

\section{Author details}

${ }^{1}$ Mistra Council for Evidence-Based Environmental Management, Royal Swedish Academy of Sciences, P.O. Box 50005, SE-104 05 Stockholm, Sweden. University of Wisconsin Center for Limnology, 680 North Park Street, Madison, WI 53706-1492, USA. ${ }^{3}$ Department of Aquatic Resources, Swedish University of Agricultural Sciences, Skolgatan 6, SE-742 42 Öregrund, Sweden. ${ }^{4}$ School of Natural Sciences, Linnaeus University, SE-391 82 Kalmar, Sweden. ${ }^{5}$ Department 
of Ecology and Environmental Science, Umeå University, SE-901 87 Umeå, Sweden. ${ }^{6}$ DTU Aqua, National Institute of Aquatic Resources, Technical University of Denmark, Vejlsøvej 39, DK-8600 Silkeborg, Denmark. ${ }^{7}$ University Museum, Norwegian University of Science and Technology, NO-7491 Trondheim, Norway. ${ }^{8}$ Department of Aquatic Ecology, Netherlands Institute of Ecology, P.O. Box 50, 6700 AB Wageningen, The Netherlands.

\section{Received: 2 December 2014 Accepted: 6 February 2015} Published online: 22 May 2015

\section{References}

1. Schindler DW. Eutrophication and recovery in experimental lakes: Implications for lake management. Science. 1974;184:897-9.

2. Jeppesen $E$, Søndergaard M, Søndergaard M, Christoffersen K. The structuring role of submerged macrophytes in lakes. In: Caldwell MM et al., editors. Ecological studies, vol. 131. New York: Springer; 1998.

3. Brönmark C, Hansson L-A. The biology of lakes and ponds. 2nd ed. Oxford: Oxford University Press; 2005.

4. Søndergaard M, Jensen JP, Jeppesen E. Role of sediment and internal loading of phosphorus in shallow lakes. Hydrobiologia. 2003;506-509:135-45.

5. Carpenter SR. Regime shifts in lake ecosystems. Oldendorf-Luhe, Germany: Ecology Institute; 2003.

6. Gårdmark A, Casini M, Huss M, van Leeuwen A, Hjelm J, Persson L, et al. Regime shifts in exploited marine food webs: detecting mechanisms underlying alternative stable states using size-structured community dynamics theory. Phil Trans R Soc B. 2014;370:20130262.

7. Søndergaard M, Jeppesen E, Lauridsen TL, Skov C, Van Nes EH, Roijackers R, et al. Lake restoration: successes, failures and longterm effects. J Appl Ecol. 2007:44:1095-105.

8. Gulati RD, Pires LMD, Van Donk E. Lake restoration studies: failures, bottlenecks and prospects of new ecotechnological measures. Limnologica. 2008;38:233-47.

9. Meijer M-L, Jeppesen E, Van Donk E, Moss B, Scheffer M, Lammens E, et al. Long-term responses to fish-stock reduction in small shallow lakes: Interpretation of five-year results of four biomanipulation cases in The Netherlands and Denmark. Hydrobiologia. 1994;275/276:457-66.

10. Perrow MR, Meijer M-L, Dawidowicz P, Coops H. Biomanipulation in shallow lakes: State of the art. Hydrobiologia. 1997;342/343:355-65.

11. Meijer M-L, de Boois I, Scheffer M, Portielje R, Hosper H. Biomanipulation in shallow lakes in the Netherlands: an evaluation of 18 case studies. Hydrobiologia. 1999:408/409:13-30.

12. Jeppesen E, Meerhoff M, Jacobsen BA, Hansen RS, Søndergaard M, Jensen JP, et al. Restoration of shallow lakes by nutrient control and biomanipulation the successful strategy varies with lake size and climate. Hydrobiologia. 2007;581:269-85

13. Bergman E, Hansson L-A, Persson A, Strand J, Romare P, Enell M, et al. Synthesis of theoretical and empirical experiences from nutrient and cyprinid reductions in Lake Ringsjön. Hydrobiologia. 1999;404:145-56.

14. Persson L. Asymmetries in competitive and predatory interactions in fish populations. In: Ebenman B, Persson L, editors. Size-structured populations: ecology and evolution. Heidelberg: Springer Verlag; 1988. p. 203-18.

15. Diehl S. Foraging efficiency of 3 fresh-water fishes - effects of structural complexity and light. Oikos. 1988;53:207-14.

16. Søndergaard $M$, Liboriussen $L$, Pedersen $A R$, Jeppesen $E$. Lake restoration by fish removal: short- and long-term effects in 36 Danish lakes. Ecosystems. 2008;11:1291-305.

17. Scheffer M, Carpenter SR. Catastrophic regime shifts in ecosystems: linking theory and observation. Trends Ecol Evol. 2003;18:648-56.

18. Carpenter SR, Ludwig D, Brock WA. Management of eutrophication for lakes subject to potentially irreversible change. Ecol Appl. 1999;9:751-71.

19. Carpenter SR. Transmission of variance through lake food webs. In: Carpenter SR, editor. Complex interactions in lake communities. New York: Springer Verlag; 1988. p. 119-35.

20. Persson L, De Roos AM. Mixed competition-predation: potential vs. realized interactions. J Animal Ecol. 2012;81:483-93.

21. Pijanowska J, Prejs A. Food-web manipulation in shallow, eutrophic lakes: Bridging the gap between the whole-lake approach and behavioural and demographic studies. Hydrobiologia. 1997;342:305-10.
22. Van de Bund WJ, Van Donk E. Short- and long-term effects of zooplanktivorous fish removal in Lake Zwemlust: a synthesis of 15 years of data. Freshw Biol. 2002;47:2380-7.

23. Shapiro J, Wright DI. Lake restoration by biomanipulation: Round Lake, Minnesota, the first two years. Freshw Biol. 1984;14:371-83.

24. Skov C, Nilsson PA. Evaluating stocking of YOY pike Esox lucius as a tool in the restoration of shallow lakes. Freshw Biol. 2007;52:1834-45.

25. Mehner T, Arlinghaus R, Berg S, Dörner H, Jacobsen L, Kasprzak P, et al. How to link biomanipulation and sustainable fisheries management: a step-by-step guideline for lakes of the European temperate zone. Fish Manag Ecol. 2004;11:261-75.

26. Liboriussen L, Søndergaard M, Jeppesen E. Sørestaurering i Danmark Del 1: Tværgående analyser, Faglig rapport fra DMU, vol. 636. Aarhus: Danmarks Miljøundersøgelser, Aarhus Universitet; 2007.

27. Olin M, Rask M, Ruuhijärvi J, Keskitalo J, Horppila J, Tallberg P, et al. Effects of biomanipulation on fish and plankton communities in ten eutrophic lakes of southern Finland. Hydrobiologia. 2006;553:67-88.

28. Jeppesen E, Søndergaard M, Lauridsen TL, Davidson TA, Liu Z, Mazzeo N, et al. Biomanipulation as a restoration tool to combat eutrophication: Recent advances and future challenges. Adv Ecol Res. 2012;47:411-88.

29. Hansson L-A, Annadotter H, Bergman E, Hamrin SF, Jeppesen E, Kairesalo T, et al. Biomanipulation as an application of food-chain theory: constraints, synthesis, and recommendations for temperate lakes. Ecosystems. 1998;1:558-74.

30. Drenner RW, Hambright KD. Biomanipulation of fish assemblages as a lake restoration technique. Archiv für Hydrobiologie. 1999;146:129-65.

31. Hansson L-A. Kan Östersjön restaureras? Utvärdering av erfarenheter från sjöar, Rapport, vol. 5860. Stockholm: Swedish Environmental Protection Agency; 2008.

32. EC. Directive $2000 / 60 / E C$ of the European Parliament and of the Council establishing a framework for the Community action in the field of water policy. 2000.

33. River Basin District Authority for the Northern Baltic Sea. Övergödda havsvikar och kustnära sjöar inom Norra Östersjöns vattendistrikt, Rapport, vol. 2009:5. Västerås: Länsstyrelsen Västmanlands län; 2009.

34. River Basin District Authority for the Southern Baltic Sea. Inventering av behovet av och möjligheterna till restaurering av övergödda havsvikar och kustnära sjöar. Kalmar: Länsstyrelserna; 2008.

35. Pullin AS, Stewart GB. Guidelines for systematic review in conservation and environmental management. Conserv Biol. 2006;20:1647-56.

36. Bernes C, Carpenter SR, Gårdmark A, Larsson P, Persson L, Skov C, et al. What is the influence on water quality in temperate eutrophic lakes of a reduction of planktivorous and benthivorous fish? A systematic review protocol. Environ Evid. 2013;2:9.

37. Lammens EHRR, van Nes EH, Meijer M-L, van den Berg MS. Effects of commercial fishery on the bream population and the expansion of Chara aspera in Lake Veluwe. Ecol Model. 2004;177:233-44.

38. Nicholls KH. Evidence for a trophic cascade effect on north-shore western Lake Erie phytoplankton prior to the zebra mussel invasion. J Great Lakes Res. 1999;25:942-9.

39. Collaboration for Environmental Evidence. Guidelines for systematic review and evidence synthesis in environmental management, vol. Version 4.2. 2013. p. 37. Environmental Evidence: www.environmentalevidence.org/ Documents/Guidelines/Guidelines4.2.pdf.

40. Landis JR, Koch GG. The measurement of observer agreement for categorical data. Biometrics. 1977;33(1):159-74.

41. Hijmans RJ, Cameron SE, Parra JL, Jones PG, Jarvis A. Very high resolution interpolated climate surfaces for global land areas. Int J Climatol. 2005;25:1965-78.

42. Viechtbauer W. Conducting meta-analyses in $\mathrm{R}$ with the metafor package. J Stat Software. 2010;36:1-48.

43. R Core Team. R: A language and environment for statistical computing. Vienna: R Foundation for Statistical Computing; 2013. http://www.R-project.org/.

44. Burnham KP, Anderson DR. Model selection and multimodel inference: a practical information-theoretic approach. New York: Springer; 2002.

45. Gulati R, van Donk E. Lakes in the Netherlands, their origin, eutrophication and restoration: state-of-the-art review. Hydrobiologia. 2002;478:73-106.

46. Mehner T, Benndorf J, Kasprzak P, Koschel R. Biomanipulation of lake ecosystems: successful applications and expanding complexity in the underlying science. Freshw Biol. 2002;47:2453-65. 